ARTICLE

DOI: $10.1038 / \mathrm{s} 41467-017-02512-1$

\title{
Trithorax dependent changes in chromatin landscape at enhancer and promoter regions drive female puberty
}

\author{
Carlos A. Toro (1) ${ }^{1}$, Hollis Wright ${ }^{1}$, Carlos F. Aylwin' ${ }^{1}$, Sergio R. Ojeda ${ }^{2}$ \& Alejandro Lomniczi (i) ${ }^{1}$
}

Polycomb group ( $\mathrm{PcG}$ ) proteins control the timing of puberty by repressing the Kiss1 gene in hypothalamic arcuate nucleus (ARC) neurons. Here we identify two members of the Trithorax group (TrxG) of modifiers, mixed-lineage leukemia 1 (MLL1), and 3 (MLL3), as central components of an activating epigenetic machinery that dynamically counteracts PcG repression. Preceding puberty, MLL1 changes the chromatin configuration at the promoters of Kiss1 and Tac3, two genes required for puberty to occur, from repressive to permissive. Concomitantly, MLL3 institutes a chromatin structure that changes the functional status of a Kiss1 enhancer from poised to active. RNAi-mediated, ARC-specific MII1 knockdown reduced Kiss1 and Tac3 expression, whereas CRISPR-Cas9-directed epigenome silencing of the Kiss1 enhancer selectively reduced Kiss1 activity. Both interventions delay puberty and disrupt reproductive cyclicity. Our results demonstrate that an epigenetic switch from transcriptional repression to activation is crucial to the regulatory mechanism controlling the timing of mammalian puberty.

\footnotetext{
${ }_{1}^{1}$ Primate Genetics Section/Division of Neuroscience, Oregon National Primate Research Center/Oregon Health and Science University, 505 NW 185 th Ave, Beaverton, OR 97006, USA. ${ }^{2}$ Division of Neuroscience, Oregon National Primate Research Center/Oregon Health and Science University, 505 NW 185 th Ave, Beaverton, OR 97006, USA. Correspondence and requests for materials should be addressed to S.R.O. (email: ojedas@ohsu.edu)

or to A.L. (email: lomniczi@ohsu.edu)
} 
T he first endocrine manifestation of the initiation of puberty is a diurnal increase in pulsatile luteinizing hormone $(\mathrm{LH})$ secretion from the pituitary gland ${ }^{1}$. This change is driven by an increase in episodic gonadotropin hormone-releasing hormone $(\mathrm{GnRH})$ release from neurosecretory hypothalamic neurons. A primary transsynaptic mechanism underlying pulsatile GnRH release involves a specialized subset of neurons located in the arcuate nucleus (ARC) of the medial basal hypothalamus $(\mathrm{MBH})^{2,3}$. They have been termed KNDy neurons, because they produce kisspeptin, neurokinin B (NKB) and dynorphin ${ }^{2,4}$. KNDy neurons release NKB, which acts on other KNDy neurons via specific receptors to stimulate kisspeptin release ${ }^{2,4}$. NKB and kisspeptin are released periodically, an oscillatory behavior determined by a phase-delayed inhibitory feedback of dynorphin on NKB release ${ }^{2,4}$. It is now clear that this periodicity underlies pulsatile LH release $\mathrm{s}^{5}$, and that both kisspeptin and NKB are required for puberty to occur. Inactivating mutations of either Kiss1 (encoding kisspeptin) or Tac3 (encoding NKB) result in hypogonadotropic hypogonadism and pubertal failure in humans $s^{6,7}$. Although there is a population of kisspeptin neurons located in the anteroventral periventricular nucleus (AVPV) of rodents [reviewed in ref. ${ }^{8}$ ], they do not contribute to the control of pulsatile GnRH release. Instead, AVPV neurons are required for the preovulatory surge of gonadotropins ${ }^{8}$. Accordingly, they do not appear to be involved in the initiation of female puberty, because the gonadotropin surge occurs only after the pubertal process is well underway.

In females, the secretory activity of KNDy neurons is kept in check during prepubertal development by an epigenetic mechanism of transcriptional repression ${ }^{9}$, exerted by the Polycomb group (PcG) of transcriptional silencers ${ }^{10}$. PcG proteins repress the Kiss1 gene to prevent the premature initiation of the pubertal process ${ }^{9}$, and this effect takes place against a backdrop of repressive chromatin, that is, rich in histone modifications associated with gene repression (such as H3K27me3 and H3K9me3) and depleted of histone modifications associated with gene activation (such as $\mathrm{H} 3 \mathrm{~K} 4 \mathrm{me} / 3$ and $\mathrm{H} 3 \mathrm{~K} 9,14 \mathrm{ac})^{11}$. During embryonic development, PcG-dependent gene repression is counterbalanced by the Trithorax group (TrxG) of transcriptional activators. Together, PcG and TrxG proteins play a major, evolutionarily conserved, role in the epigenetic control of gene expression required for the establishment of cellular diversity ${ }^{12}$. TrxG proteins counteract the effect of PcG proteins by methylating histone 3 at lysine $4(\mathrm{H} 3 \mathrm{~K} 4)^{13,14}$, a modification that establishes a permissive chromatin configuration, i.e., enriched in $\mathrm{H} 3 \mathrm{~K} 4 \mathrm{me} / 3$ and $\mathrm{H} 3 \mathrm{~K} 9,14 \mathrm{ac}$, with or without loss of H3K27me3 and $\mathrm{H} 3 \mathrm{~K} 9 \mathrm{me} 3^{15,16}$. In mammals, there are six TrxG protein complexes termed COMPASS (Complex of Proteins associated to Set1) and COMPASS-like, because they are related to the original yeast SET1 methyltransferase ${ }^{13,14}$. Two of these complexes (SET1A and SET1B COMPASS) contain Drosophila SET1-related proteins; two (known as COMPASS-like) contain the proteins MLL1/KMT2A or MLL2/KMT2B, which are related to Drosophila Trithorax (Trx), and the other two (also termed COMPASSlike) contain either MLL3/KMT2C or MLL4/KMT2D, both of them related to Drosophila Trr (Trx-related). While SET1A/ SET1B and MLL1/MLL2 mediate H3K4 trimethylation (H3K4me3) at promoters ${ }^{17,18}$, MLL3/MLL4 catalyze monomethylation of H3K4 (H3K4mel) at enhancer sites ${ }^{19}$.

It has been shown that as the PcG repressive influence wanes before puberty, the abundance of histone modifications either catalyzed by (H3K4me3), or associated to $(\mathrm{H} 3 \mathrm{~K} 9,14 \mathrm{ac})$ the Trithorax group (TrxG) of transcriptional activators ${ }^{13}$ increases at the Kiss1 promoter $^{9}$, implicating the TrxG complex as the facilitatory counterpart of PcG-mediated gene silencing. Should these interactions also occur at the Tac3 promoter, it would strongly suggest that the epigenetic control of KNDy neurons, and hence the pubertal process itself, is a coordinated process involving the two principal gene systems that, operating within the ARC, spur reproductive development. The presence of both repressive and stimulatory epigenetic pathways regulating Kiss 1 and Tac3 expression in KNDy neurons suggests that a switch from epigenetic repression to activation within these neurons underlies the developmental process by which GnRH release increases by late juvenile development to bring about the pubertal process. In a broader context, it also raises the tantalizing possibility that mammalian puberty might be a major developmental milestone regulated by the counterbalancing actions of the PcG and TrxG complexes operating within the neuroendocrine brain. The present study provides experimental evidence supporting this concept.

\section{Results}

TrxG gene expression in the MBH during pubertal development. We used massively parallel sequencing (RNA-seq) to determine if the advent of female puberty is preceded by changes in $\mathrm{MBH}$ expression of any of the 18 genes known to be components of the TrxG complex. We examined these changes in female rats during juvenile development (postnatal days (PND) 21-28), i.e., at the time when expression of Eed and Cbx7, two key members of the PcG complex, declines ${ }^{9}$. Only Mll1, a member of one of the COMPASS-like family of TrxG genes ${ }^{12}$ (Fig. 1a), showed increased expression between the beginning (EJ/PND21) and the end (LJ/PND28) of the juvenile period (Fig. 1b). A more detailed quantitative PCR (qPCR) examination of TrxG gene expression before and at the completion of puberty revealed that Mll1 mRNA levels remain elevated on the day when the first preovulatory surge of gonadotropins takes place (Fig. 1c), i.e., at the completion of puberty. Though less noticeable, expression of other TrxG genes also increases either before (Set1a and Utx) or at the time of puberty (Set1b, Mll3, and Dpy30). Set1a and Set1b encode methyltransferases responsible for the bulk di- and trimethylation of lysine 4 at histone 3 across the genome ${ }^{13,14}$. The MBH from female rhesus monkeys undergoing puberty exhibited an increase in expression of MLL1, SET1A, and DPY30 similar to that detected in rats (Supplementary Fig. 1), indicating that puberty-related changes in hypothalamic TrxG expression are not limited to rodents, but also occur in nonhuman primates. Using double fluorescent in situ hybridization (FISH) we observed that cells expressing Mll1 mRNA were abundant in the ARC (Fig. 2a, b) and that within this region KNDy neurons, identified by the presence of Kiss1 mRNA, contain Mll1 mRNA transcripts (Fig. 2c-e). KNDy neurons also express Mll3 (Fig. $2 \mathrm{~h}-\mathrm{j}$ ), a member of the COMPASS family that activates distal enhancer sites by monomethylating histone $\mathrm{H} 3 \mathrm{~K} 4$, promoting $\mathrm{H} 3 \mathrm{~K} 27$ acetylation, and antagonizing PcG silencing ${ }^{19,20}$. In addition, KNDy neurons express other key components of the TrxG complex, including Set1b (Supplementary Fig. 2), Mll2 (Supplementary Fig. 3), and Mll4 (Supplementary Fig. 4). MLL4 stimulates enhancer activity by eliciting changes in histone configuration similar to those catalyzed by MLL3 ${ }^{19,20}$. These results demonstrate that KNDy neurons express all members of the TrxG activating complex required to counteract $\mathrm{PcG}$ silencing, and epigenetically enhance the transcription of genes involved in the stimulatory control of puberty.

Recruitment of TrxG proteins to puberty-activating genes. We next used chromatin immunoprecipitation (ChIP)-qPCR assays to determine if recruitment of core COMPASS-like proteins to the promoter region of Kiss1, Tac3, and Pdyn, the genetic 
backbone of KNDy neuron reproductive function, changes in the $\mathrm{MBH}$ during the days antedating puberty. We focused our attention to COMPASS-like complexes containing MLL1-MLL4 instead of SET1A or SET1B, because MLL1-MLL4-dependent $\mathrm{H} 3 \mathrm{~K} 4$ methylation is an event that affects a selected population of genes involved in regulating discrete and specialized functions, in contrast to SET1A and SET1B, which are responsible for the global, genome-wide, di- and trimethylation of $\mathrm{H} 3 \mathrm{~K} 4^{13,14}$. Regulation of Kiss 1 and Tac3 gene expression in the ARC by TrxG proteins in the context of pubertal development is, undoubtedly, one of these specialized processes.
Both Kiss 1 and Tac3 mRNA levels begin to increase in the ARC at the initiation of juvenile development (EJ) with respect to the infantile phase (INF/PND14), becoming even more elevated by the LJ period, i.e., at the initiation of puberty (Fig. 3a). In contrast, $P d y n$ expression remained unchanged during the entire prepubertal period (Fig. 3a). Consistent with the concept of a coordinated epigenetic input regulating the stimulatory arm of KNDy neurons, we observed that the abundance of MLL1 at both the Kiss 1 and Tac3 promoters increases concomitantly with the change in gene expression (Fig. 3b). MLL2 association to the Kiss1 promoter did not change, but increased modestly at a

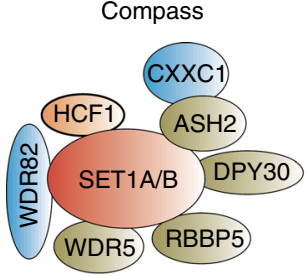

Compass-like

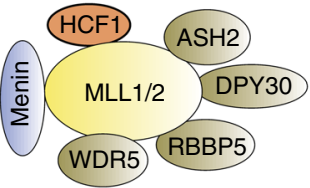

Compass-like

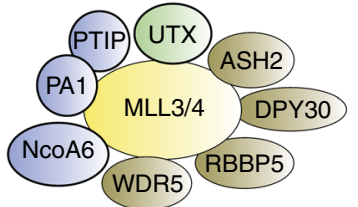

\section{b}

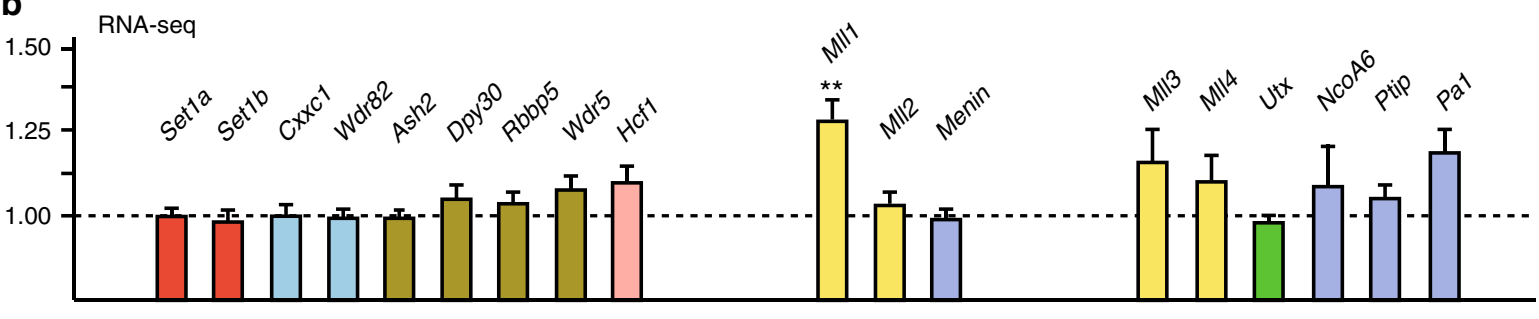

Fold change EJ-LJ (PND21-PND28)

C
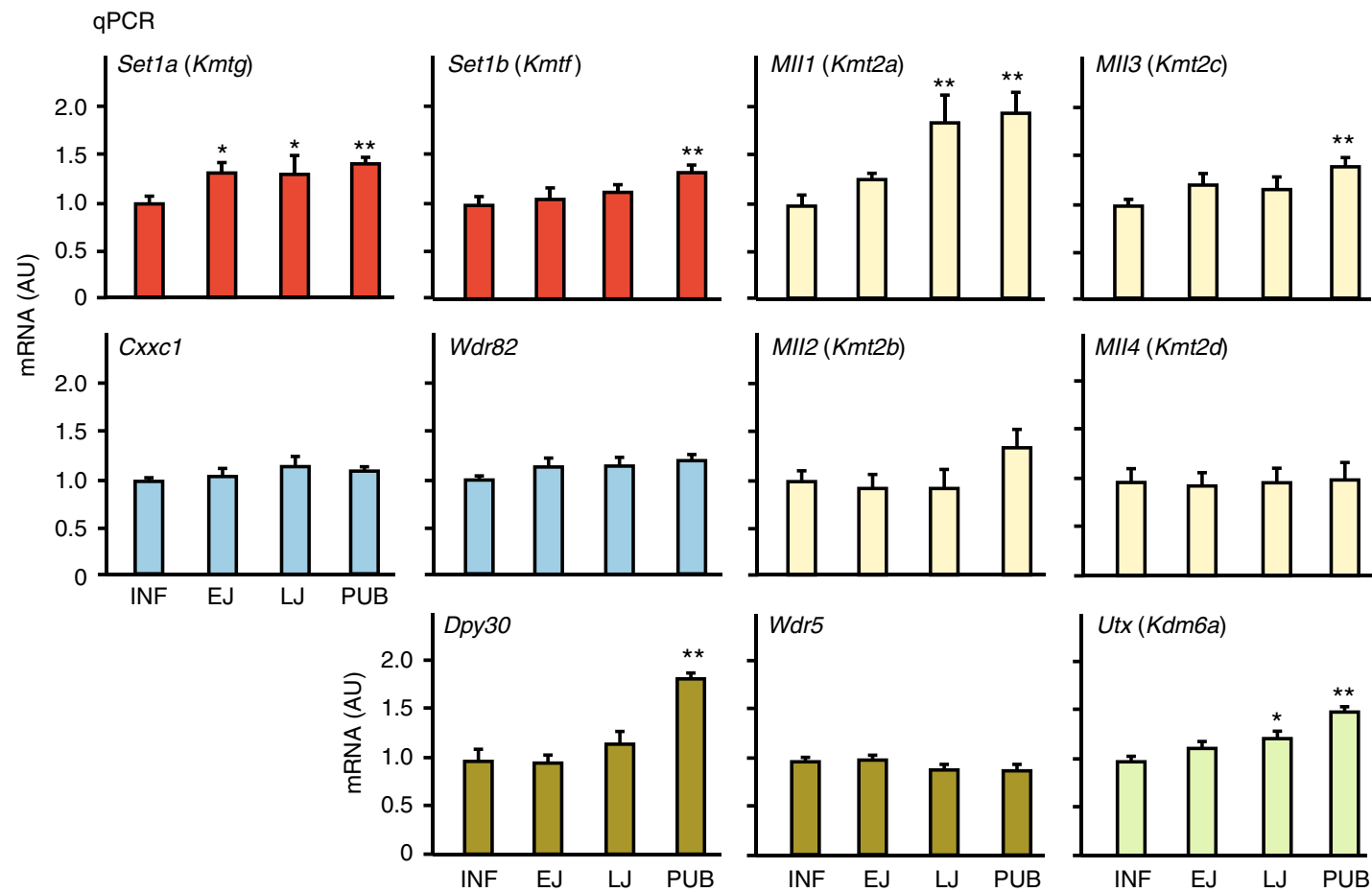

Fig. 1 TrxG gene expression in the prepubertal female rat hypothalamus. a The COMPASS families (for details see Introduction). b Expression of COMPASS genes in the MBH of prepubertal female rats assessed by RNA-seq. Data are represented as fold change between the early juvenile and the late juvenile groups (EJ/LJ). Dotted line depicts the EJ/LJ ratio of 1. Bars represent mean \pm s.e.m. $(n=4) .{ }^{\star} P<0.01$ vs. EJ group; Student's $t$-test. c Expression of COMPASS genes in the MBH of peripubertal female rats as determined by qPCR. RNA expression data were normalized using peptidylprolyl isomerase $A$ as the housekeeping gene and by dividing each individual value by the average of the INF group. Bars represent mean \pm s.e. $\mathrm{m}$. INF $=$ infantile $(n=7-8)$; EJ $=$ early juvenile $(n=5-7) ; \mathrm{LJ}=$ late juvenile $(n=6-8) ; \mathrm{PUB}=$ pubertal $(n=6-9)$, day of the first preovulatory surge of gonadotropins. ${ }^{\star} P<0.05 ;$ and ${ }^{\star \star} P<$ 0.01 vs. INF; one-way ANOVA followed by Student-Newman-Keuls (SNK) test 
the Tac3 promoter at LJ (Supplementary Fig. 5a). In contrast, recruitment of both MLL1 and MLL2 to the Pdyn promoter remained low and unaltered throughout the entire postnatal period studied (Fig. 3b and Supplementary Fig. 5a). Assessment of MLL3 recruitment revealed an increase in MLL3 association to both the Kiss 1 and Tac3 promoters at LJ, with no changes detected at the Pdyn promoter (Fig. 3c). In contrast to MLL3, MLL4 association to each of these three promoters remained unaltered (Supplementary Fig. 5b). Concordant with the increase in MLL1 association to the Kiss1 and Tac3 promoters, H3K4me2 abundance at both promoters (but not at the $P d y n$ promoter) increased strikingly between the INF and EJ periods, remaining elevated thereafter (Fig. 3d). A similar strong increase in H3K4me3 content was observed at both the Kiss1 and Tac3 promoters, but instead of occurring at EJ, it became apparent at LJ, Fig. 3e), when puberty is initiated. These changes were hypothalamic-specific, because they were not observed in the cerebral cortex (CTX; insets in Fig. 3e). They were correlated with increases in both Kiss 1 and Tac3 mRNA levels that began at EJ, when H3K4me2 content was elevated, and became maximal at LJ, coinciding with the prepubertal increase in $\mathrm{H} 3 \mathrm{~K} 4 \mathrm{me} 3$ content (Fig. 3a).

In contrast to TrxG proteins and associated histone marks, EED — a PcG protein required for PcG-mediated gene silencing- was evicted from the Kiss 1 and Tac3 promoters during juvenile development (EJ-LJ; Fig. 3f). As shown before ${ }^{9}$, depletion of H3K27me3 (the histone modification catalyzed by the PcG complex) did not occur at this time (Fig. 3g). Instead, H3K27me3 content decreased at the late proestrus (LP) stage (insets in Fig. 3g), the phase of puberty when the first preovulatory surge of gonadotropins takes place. The coexistence of the permissive/ activating marks $\mathrm{H} 3 \mathrm{~K} 4 \mathrm{me} 2$ and $\mathrm{H} 3 \mathrm{~K} 4 \mathrm{me} 3$ with the repressive H3K27me3 modification is a feature of "bivalent" promoters (i.e., promoters of genes poised for activation in response to incoming stimuli) ${ }^{21}$. Based on these features, the Kiss 1 promoter has been postulated to be bivalent ${ }^{9}$.

Contrary to Kiss 1 and Tac3, H3K27me3 content at the Pdyn promoter increased during juvenile development (Fig. 3g). Although this change implies the institution of a PcGdependent repression of $P d y n$ transcription at puberty, the lack of concomitant changes in EED recruitment to the Pdyn promoter (Fig. 3f) makes this inference less certain. Both the Kiss1 and Tac3 promoters appear to be subjected to some PcGindependent repressive control, as suggested by the mild loss of $\mathrm{H} 3 \mathrm{~K} 9 \mathrm{me}$, a repressive mark deposited by the histone methyltransferase SETDB1 ${ }^{22}$, from both the Kiss 1 and Tac3 promoters at LJ (Fig. 3h). Overall, these results indicate that a dynamic balance between the PcG and TrxG complexes regulating Kiss1
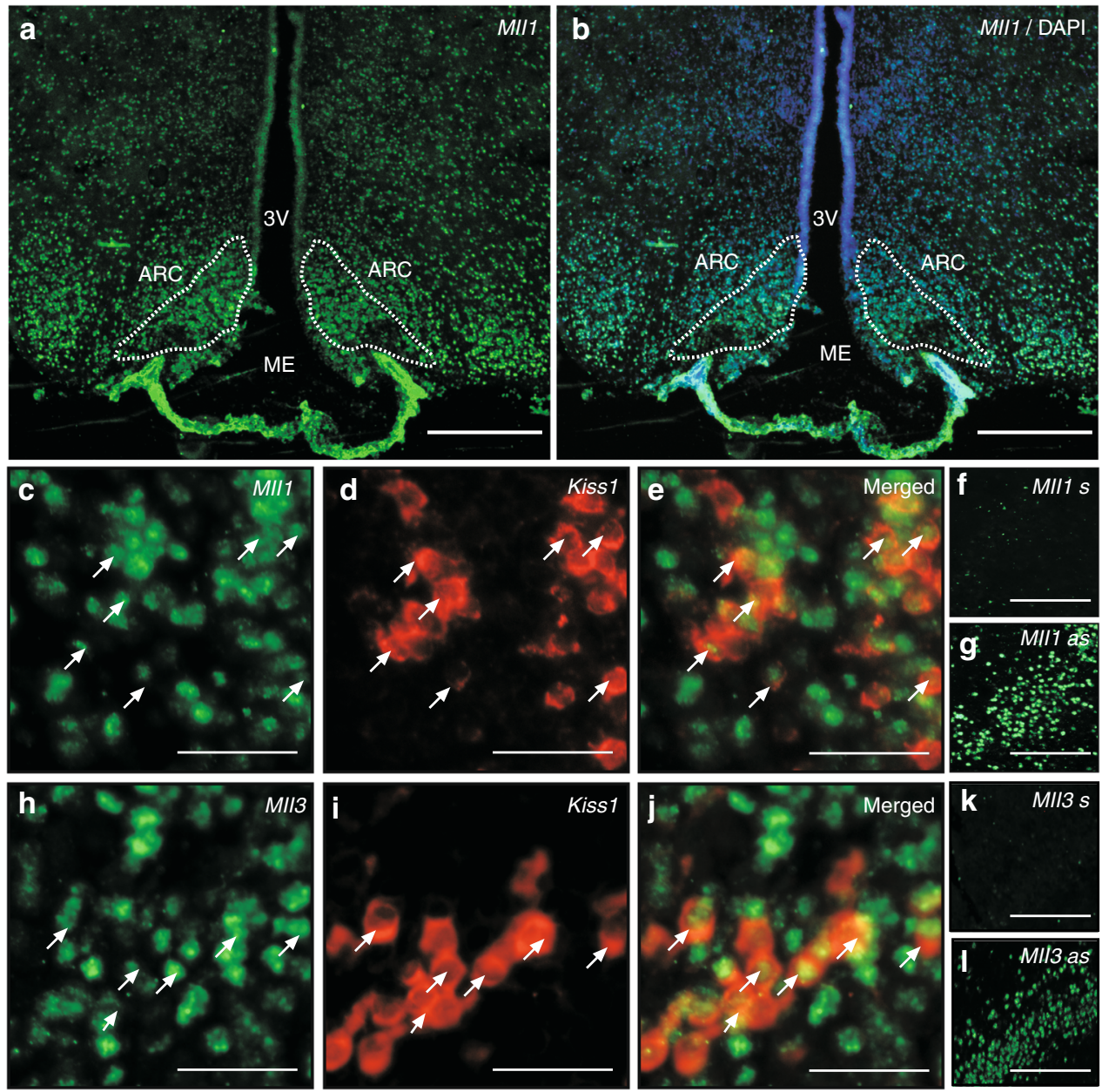

Fig. 2 MII1 and MII3 mRNA transcripts in KNDy neurons of late juvenile female rats. a, b Abundance of MII1 mRNA transcripts (green color) in the arcuate nucleus (ARC, dotted line) region of the $\mathrm{MBH}$, as determined by FISH. 3V, third ventricle; ME, median eminence. Bars, $500 \mu \mathrm{m}$. c-e KNDy neurons of the ARC contain MII1 mRNA as determined by double FISH (Kiss1 mRNA, red; MII1 mRNA, green). h-j KNDy neurons also contain MII3 mRNA. Bars, 100 $\mu$ m. $\mathbf{f}, \mathbf{g}$ Lack of hybridization in sections incubated with a sense MII1 RNA probe. $\mathbf{k}$, I Lack of hybridization in sections incubated with a sense MII3 RNA probe. Bars, $200 \mu \mathrm{m}$. $\mathrm{s}=$ sense; as = antisense 
and Tac3 expression in the ARC is a central feature of the epigenetic control of puberty

MLL1 trans-activates puberty-activating genes. Consistent with this concept, MLL1 enhanced Kiss1 and Tac3, but not Pdyn, transcriptional activity in gene promoter assays, and EED abolished this effect (Fig. 4). MLL1 also enhanced the transcriptional activity of Ttf1, Eap1, and Nell2 (Supplementary Fig 6a), a set of genes involved in the stimulatory control of female puberty ${ }^{23-25}$, but did not increase the transcription of genes keeping the pubertal process in check (Eed, MKRN3, and PENK; Supplementary Fig 6b). For a trans-activating effect of MLL1 to also occur in vivo, MLL1 recruitment to these promoters would be expected to increase with the advent of puberty. Our results show that whereas MLL1 recruitment to the Kiss1 and Tac3 promoters does increase markedly at this time (Fig. 3b), it remains unchanged at the Nell2 and Ttf1 promoters (selected as representing pubertyactivating genes other than Kiss1 and Tac3; Supplementary Fig. 6c). Altogether, these findings demonstrate that MLL1 binding to the Kiss 1 and Tac3 promoters increases selectively at puberty and activates transcription of these two pubertyactivating genes, which are essential for puberty to occur.

ARC-targeted loss of Mll1 delays puberty. To determine if loss of Mll1 would affect the timing of puberty we used an RNAi approach. We generated lentiviral (LV) constructs targeting Mll1 mRNA via short hairpin (sh) RNAs inserted into the body of miRNA-30 and under the control of the U6 promoter

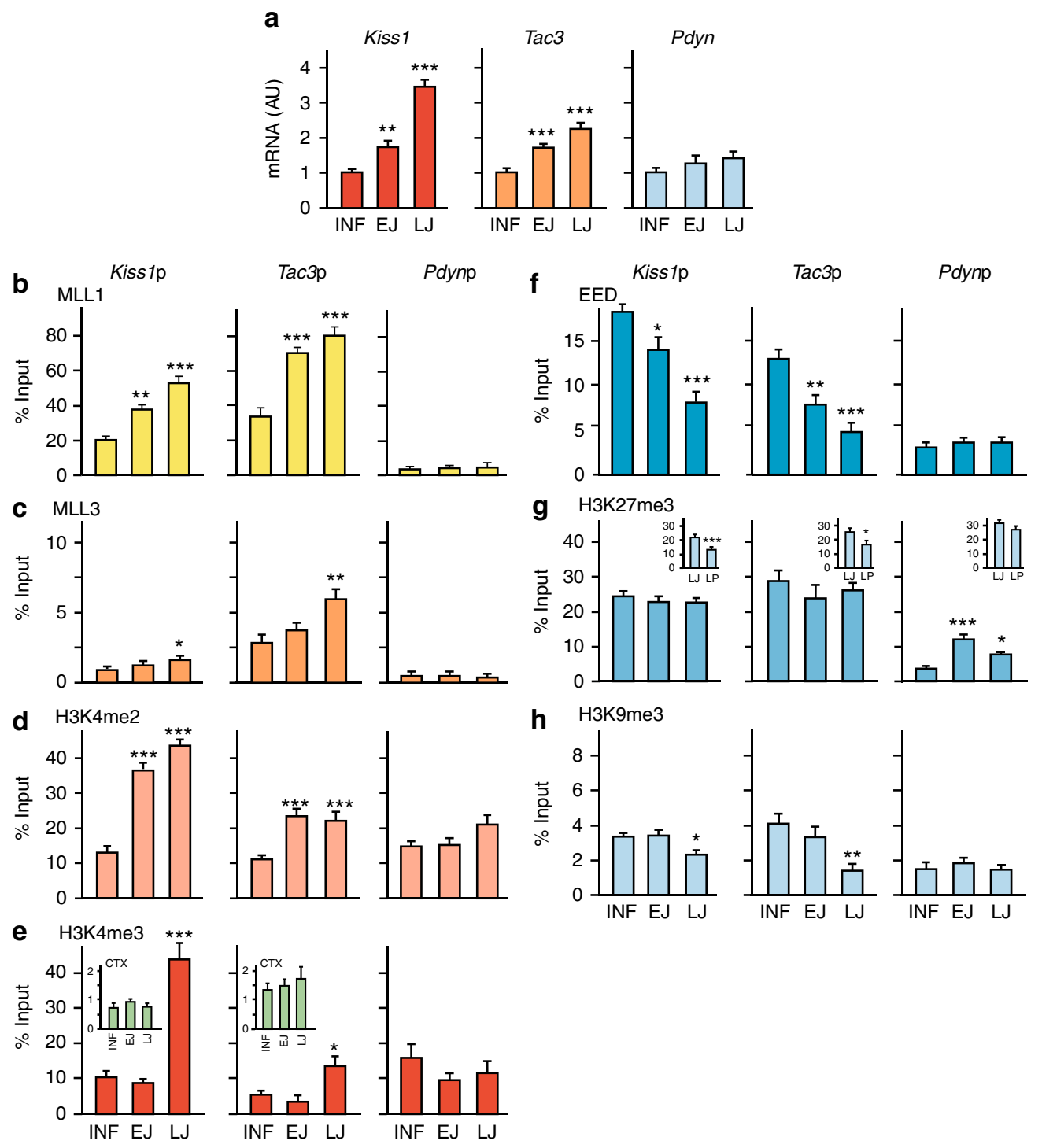

Fig. 3 Kiss1, Tac3, and Pdyn mRNA expression, and promoter chromatin state in the prepubertal female rat hypothalamus. a Expression of Kiss1, Tac3, and Pdyn mRNA in the MBH of prepubertal female rats as determined by qPCR. Bars represent mean \pm s.e.m. (INF, EJ, and LJ; $n=8$ ); $A U=$ arbitrary units. RNA expression data were normalized by dividing each individual value by the average of the INF group. $\mathbf{b}$ Changes in MLL1 recruitment to the Kiss1, Tac3, and Pdyn promoters before puberty. c Changes in MLL3 recruitment to the Kiss1, Tac3, and Pdyn promoters before puberty. d, e Abundance of the TrxGdependent activating marks H3K4me2 (d) and H3K4me3 (e) during prepubertal development. Insets in e depict H3K4me3 content at the Kiss1 and Tac3 promoters in the CTX during prepubertal development. $\mathbf{f}$ EED recruitment to the Kiss1, Tac3, and Pdyn promoters before puberty. $\mathbf{g}$ Abundance of the PcGdependent repressive mark H3K27me3 at the Kiss1, Tac3, and Pdyn before puberty or at the time of the first preovulatory surge of gonadotropins (late proestrus, LP) at the Kiss1 and Tac3 promoters (insets in $\mathbf{g}$ ). h Abundance of the PcG-independent repressive mark H3K9me3 at the Kiss1, Tac3, and Pdyn promoters before puberty. Bars represent mean \pm s.e.m. (INF, EJ, and LJ; $n=5-8$; insets in $\mathbf{e} ; n=4$; insets in $\mathbf{g} ; n=6$ ). ${ }^{\star} P<0.05$, ${ }^{\star \star} P<0.01$, and ${ }^{\star \star \star} P<$ 0.001 vs. INF; one-way ANOVA followed by SNK test 
(Supplementary Fig. 7a). Transduction of the R22 rat hypothalamic cell line with these constructs demonstrated that two of the four shRNAs tested (sh2612 and sh12751) were effective in lowering Mll1 mRNA levels (Supplementary Fig. 7b). Because the LV constructs employed encode an enhanced green fluorescent protein (eGFP) protein, we used this feature to isolate via fluorescent-activated cell sorting (FACS; Supplementary Fig. 7c) the cells transduced with the two effective shRNAs. qPCR analysis verified the effectiveness of both sh2612 and sh12751 in reducing Mll1 expression (Supplementary Fig. 7d). Both shRNAs also reduced the levels of Kiss1 and Tac3 mRNA (Supplementary Fig. 7d), without affecting Eed and Pdyn mRNAs (Supplementary Fig. 7e), suggesting that as previously shown by the results of promoter assays, MLL1 trans-activates Kiss1 and Tac3 gene expression, but not expression of puberty-inhibitory genes.

We then sought to determine if silencing of Mll1 in the ARC is capable of delaying the time of puberty. We delivered an LV construct encoding shRNA 2612 (sh2612; Fig. 5a) to the ARC of EJ female rats via bilateral microinjections. Control animals were injected with an LV construct carrying GFP (C). Animals with correctly placed sh2612 (Fig. 5b) had a significant delay in both the age at vaginal opening (VO; Fig. 5c; mean age at VO: C, $31 \pm$ 0.2 days vs. $\operatorname{sh} 2612,34.4 \pm 0.7$ days; $t=5.02, P<0.001$, Student's $t$-test) and at first estrous (Fig. $5 \mathrm{~d}$ ); mean age at first estrous: $\mathrm{C}$, $32 \pm 0.6$ days vs. sh2612, $38.3 \pm 0.6$ days; $t=7.25, P<0.001$, Student's $t$-test). The first estrous is when the first ovulation takes place $^{9}$. By the time when all control animals had shown VO, only $30 \%$ of sh2612 animals had done so (Fig. 5c). Likewise, none of the sh2612 rats had ovulated at the age when all control rats had already completed this pubertal phase (Fig. 5d). In addition, estrous cyclicity was compromised (Fig. 5e, f), with the sh2612injected animals showing fewer days in proestrus and many more days in an intermediate stage (estrous/diestrus) than C-injected rats (Fig. 5e, f).

To determine if the loss of Mll1 targeted to the ARC resulted in the expected changes in gene expression, we injected another group of EJ rats and collected the $\mathrm{MBH}$ at LJ for mRNA quantitation. In agreement with the in vitro results, animals injected with sh2612 showed a pronounced reduction in Mll1, Kiss1, and Tac3 mRNA abundance without alteration in the expression of Pdyn (Fig. 5g), the third member of the triad that, expressed in KDNy neurons, controls pubertal development. Importantly, sh2612 did not alter the expression of a number of other genes contributing to either repressing the pubertal process (Eed, Mkrn3, and pEnk; Fig. 5h) or involved in the stimulatory control of puberty (Ttf1, Nell2, Eap1, Gpr54, encoding the kisspeptin receptor; Tac $3 R$, a preferred NKB receptor; Grin $2 a$ and Grin2d, encoding NMDA-type of glutamatergic receptors; and Grik5, encoding a kainate-type of glutamatergic receptor; Fig. 5i).
The lack of a compensatory increase in Eed expression after loss of Mll1 expression (Fig. 5h) is in agreement with a previous report showing that siRNA-induced Mll1 knockdown in a different cell system does not result in increased PcG gene expression $^{26}$. Thus, prepubertal loss of Mll1 in the ARC selectively reduces Kiss 1 and Tac3 expression, compromises the initiation and completion of puberty, and disrupts post-pubertal reproductive cyclicity.

A Kiss1 enhancer is activated before puberty. Kiss 1 expression has been postulated to be controlled by two different enhancers, one ARC-specific located upstream from the transcription start site (TSS) ${ }^{27}$, and one AVPV-specific located downstream from the $3^{\prime}$-end of the gene ${ }^{28}$. Because enhancers of active genes display high levels of $\mathrm{H} 3 \mathrm{~K} 4 \mathrm{mel}$ and $\mathrm{H} 3 \mathrm{~K} 27 \mathrm{ac}^{29}$, we performed a genome-wide analysis of these two histone modifications in the $\mathrm{MBH}$ of LJ rats to identify potential enhancers of Kiss1 transcription. A $12 \mathrm{~kb}$ segment of the Kiss $15^{\prime}$ flanking region displayed two areas showing elevated levels of H3K4mel and H3K27ac, one located between nucleotides (nt) -3188 and -2086 (site 1) and a more distal region (site 2) located approximately between $n t-9400$ and -7900 (Fig. 6a). Of these, site 1 is DNase Ihypersensitive in both mice and humans (Fig. 6a) and displays a histone mark signature typical of enhancers, including the presence of p300/CBP-binding sites (Fig. 6a; https://www. encodeproject.org/). Deletion of a genomic fragment containing site 1 results in selective loss of Kiss1 expression in KNDy neurons, but not AVPV kisspeptin neurons of mice, indicating that site 1 is an ARC-specific Kiss 1 enhancer ${ }^{27}$. Consistent with this notion, analysis of site 1 and site 2 in the rat genome by targeted ChIP-qPCR revealed that only site 1 displayed significant association of p300/CBP, a hallmark of active enhancers ${ }^{29,30}$ and that the levels of both p300/CBP and H3K27ac increase significantly during prepubertal development (Fig. $6 \mathrm{~b}$ ). This demonstrates that site 1 is an enhancer that becomes active as the animal approaches puberty, and suggest that the previously postulated ARCspecific Kiss1 enhancer ${ }^{27}$ resides in this genomic region. The COMPASS-like proteins MLL3 and/or MLL4 are responsible for depositing the $\mathrm{H} 3 \mathrm{~K} 4 \mathrm{me} 1$ mark at distal enhancers and are required for p300/CBP-dependent $\mathrm{H} 3 \mathrm{~K} 27$ acetylation at these regions ${ }^{19,20}$. In keeping with this concept, MLL3 recruitment to site 1 (but not site 2) increased significantly between EJ and LJ (Fig. 6b). Noteworthy, the changes in MLL3 recruitment to site 1 appear to be specific to MLL3, as they were not accompanied by similar changes in MLL4 occupancy. Because p300/CBP is recruited to enhancer regions via physical association with MLL3 or MLL4, and requires this association to promote H3K27 acetylation $^{20}$, these results suggest that the changes in $\mathrm{p} 300 / \mathrm{CBP}$
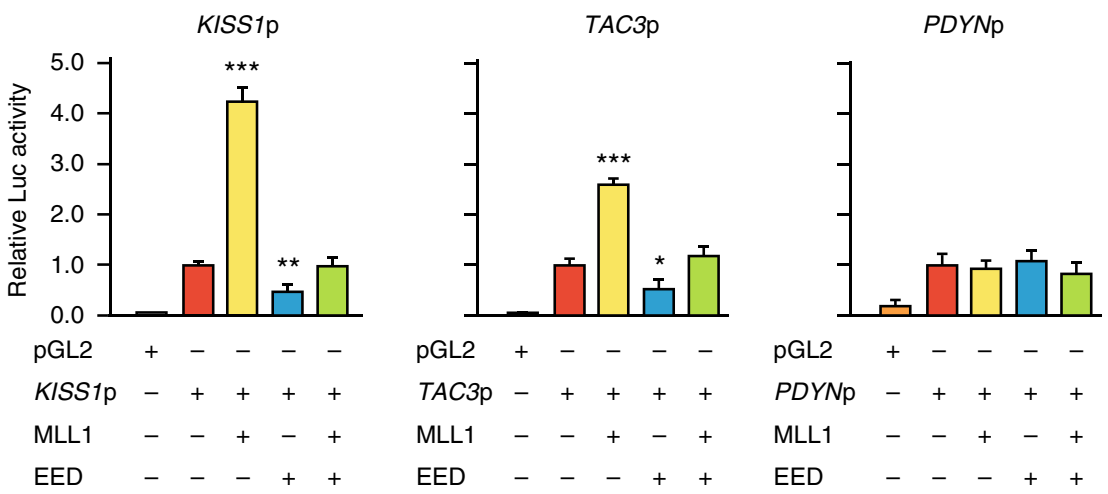

Fig. 4 Effect of MLL1 and EED on KISS1, TAC3, and PDYN promoter (p) activity. Bars represent mean \pm s.e.m. $(n=6)$. ${ }^{\star \star \star} P<0.001$ vs. all other groups; ${ }^{\star \star} P$ $<0.01$ vs. Kiss1 promoter alone; ${ }^{\star} P<0.05$ vs. Tac3 promoter alone; one-way ANOVA followed by SNK test 
a

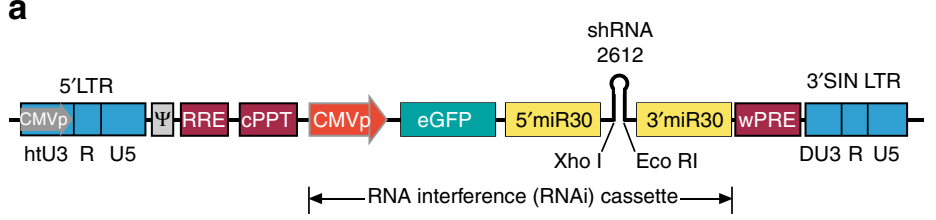

b

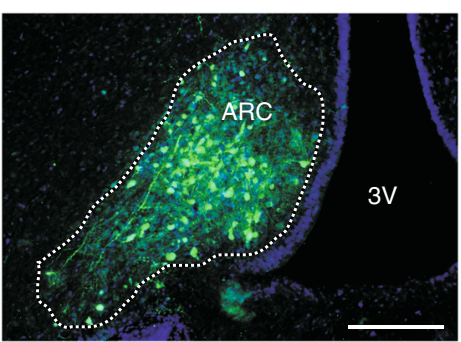

C

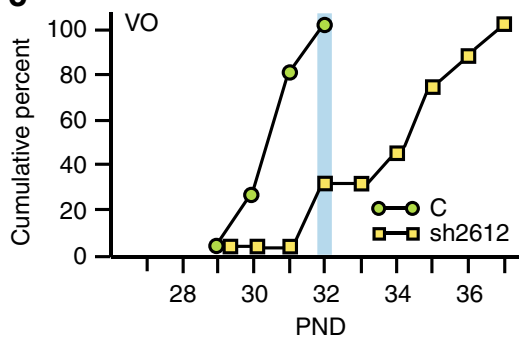

e

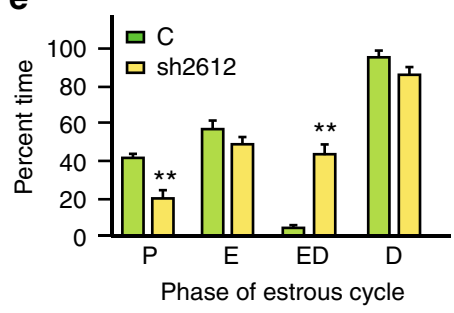

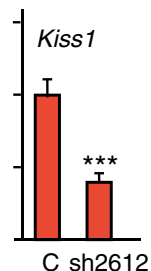
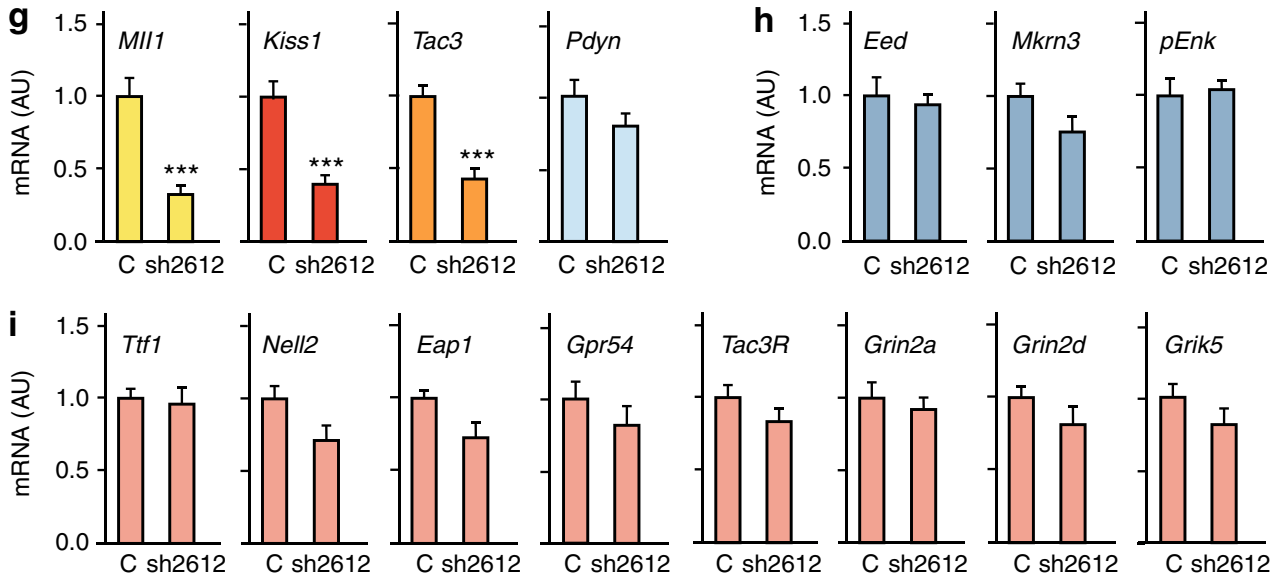

Fig. 5 Silencing the MII1 gene in the ARC of immature female rats. a Lentiviral construct (LV-shMII1-GFP) encoding sh2612 that most effectively silences MII1. b Transduction of ARC cells with sh2612 delivered to the ARC of EJ female rats. Green: sh2612 infected cells; blue: Hoechst-stained cell nuclei in the MBH; dotted line: ARC. Bars, $200 \mu \mathrm{m}$. c Cumulative percent day at vaginal opening (VO), and $\mathbf{d}$ cumulative percent day at first estrus (FE) in control (C; $n=9)$ and sh2612 ( $n=7)$-injected rats. Blue shade depicts the time when all $C$ animals show VO or FE. e Percent of time spent in different stages of the estrous cycle by rats injected with $\mathrm{C}(n=9)$ or sh2612 $(n=7)$. f Examples of estrous cycle patterns in rats injected with $\mathrm{C}$ or sh2612. g MII1, Kiss1, Tac3, and Pdyn mRNA levels detected by qPCR in the ARC of $L J$ female rats receiving $C(n=8)$ or $\operatorname{sh} 2612(n=8)$. $\mathbf{h}$ Expression of puberty-inhibitory or $\mathbf{i}$ puberty-activating genes in the ARC of LJ female rats receiving $C(n=8)$ or sh2612 $(n=8)$. RNA expression data were normalized by using peptidylprolyl isomerase A as the housekeeping gene and dividing each individual value by the average of the $C$ group. $P=$ proestrous; $E=$ estrous; $E D=$ transitional phase (estrous/ diestrous); $\mathrm{D}=$ diestrous; $\mathrm{AU}=$ arbitrary units; $3 \mathrm{~V}=$ third ventricle. Bars represent mean \pm s.e.m. $\left({ }^{\star \star} P<0.01,{ }^{\star \star \star} P<0.001\right.$ vs. $\mathrm{C}$, Student's $t$-test) 
observed at site 1 during juvenile development are MLL3dependent. TrxG-p300/CBP-mediated activation of distal enhancers counteracts PcG-mediated silencing ${ }^{20,31}$ to facilitate the transition from a poised to an active configuration ${ }^{31}$.

Poised enhancers contain an abundance of H3K4me1, low levels of the activating histone mark H3K27ac, and high levels of the PcG-dependent repressive mark H3K27me3. Active enhancers also contain $\mathrm{H} 3 \mathrm{~K} 4 \mathrm{me} 1$, but are enriched in $\mathrm{H} 3 \mathrm{~K} 27 \mathrm{ac}$ and depleted of H3K27me $3^{29,32}$. Our results show a loss of EED and H3K27me3 from site 1, but not from site 2, during juvenile development (Fig. 6b), suggesting that site 1 is an enhancer whose activity increases antedating the initiation of female puberty due to increased TrxG stimulation coupled to loss of PcG repression.

Epigenome silencing of the ARC Kiss1 enhancer. To determine if site 1 is indeed a biologically relevant ARC-specific Kiss 1 enhancer we subjected site 1 to epigenome editing using clustered regularly interspaced short palindromic repeats (CRISPR)-Cas9 technology and a catalytically inactive Cas9 (dCas9) from Staphylococcus aureus (SaCas9) fused to the Krüppel-associated box (KRAB) silencing domain from Kox1/ZNF10 $0^{33,34}$. Because dCas9 binds, but does not cleave DNA, dCas9 functions as a DNAbinding domain that is targeted to genomic regions of interest by single-guide RNAs (sgRNAs) complementary to DNA sequences adjacent to a motif known as the protospacer adjacent motif $(\mathrm{PAM})^{35}$. Since we employed dSaCas9, our sgRNAs were $22 \mathrm{nt}$ long and targeted DNA sequences adjacent to the PAM motif NNGRRT/NNGRR ${ }^{36}$. When localized to genomic DNA by this targeting platform, the KRAB repressive domain facilitates the formation of a heterochromatin complex that includes the methyltransferase SETDB1 and the deacetylase $\mathrm{HDAC1}^{37}$.

We prepared adeno-associated virus (AAV)-dSaCas9-KRABsgRNA constructs carrying five different sgRNAs targeting site 1 (Fig. 7a) and transfected these constructs into Ratl cells. Two days later, we measured $\mathrm{H} 3 \mathrm{~K} 9 \mathrm{me} 3$ (the histone mark catalyzed by SETDB1) and H3K27ac at site 1 by ChIP assays. Of the five constructs tested, the two sgRNAs targeting the $3^{\prime}$-end of site 1 (sg4 and sg5) were more effective in increasing $\mathrm{H} 3 \mathrm{~K} 9 \mathrm{me} 3$ content (Fig. 7b). In contrast, none of the sgRNAs tested affected the abundance of $\mathrm{H} 3 \mathrm{~K} 9 \mathrm{me} 3$ at the Kiss 1 promoter (Fig. 7c), indicating that sg4 and sg5 specifically established a repressive chromatin configuration at the Kiss 1 enhancer, leaving the

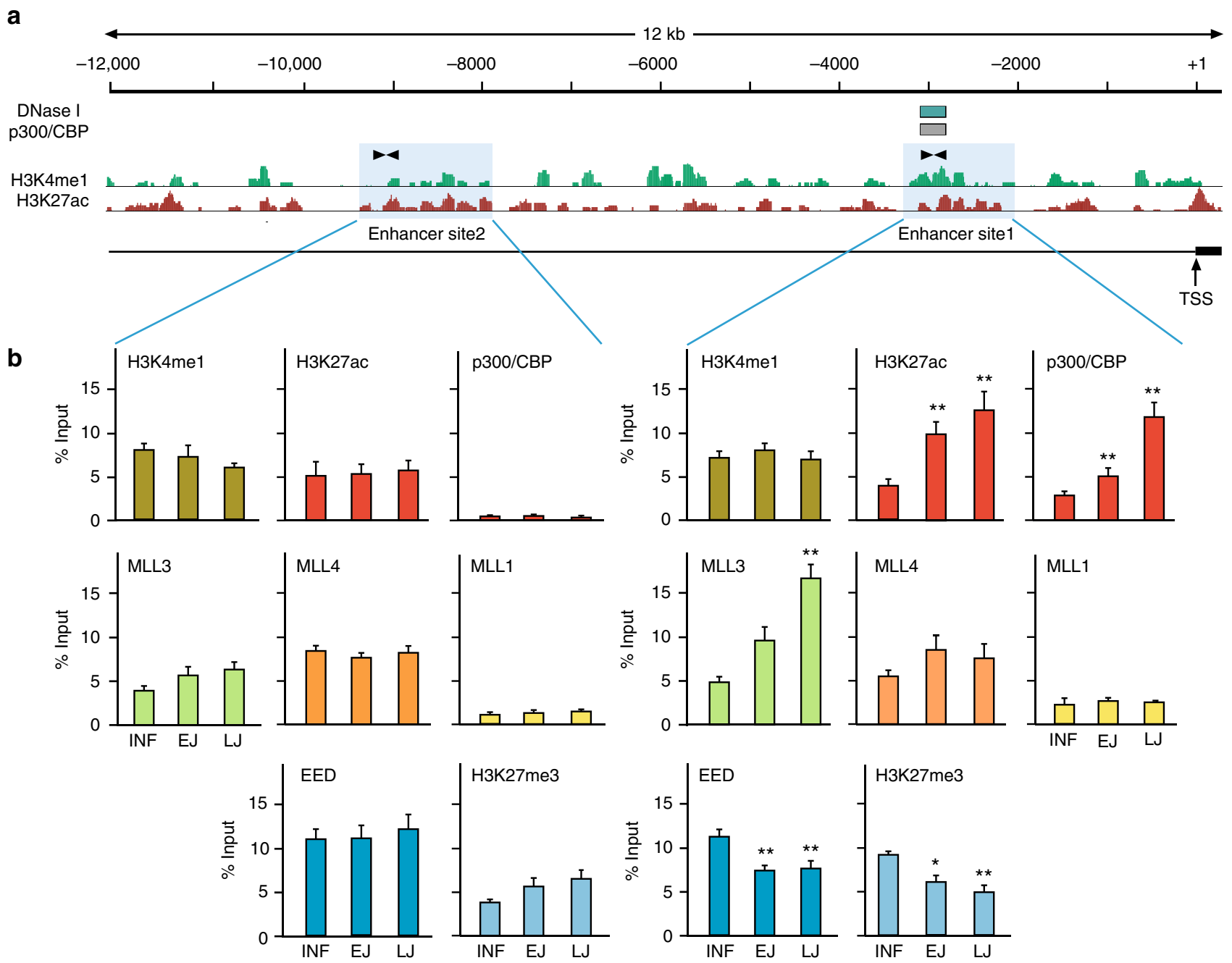

Fig. 6 A genomic region upstream of the Kiss1 gene behaves as a puberty-activated enhancer domain. a ChIP-seq tracts of H3K4me1 and H4K27ac within $12 \mathrm{~kb}$ of Kiss $15^{\prime}$-flanking region detected using ARC chromatin from LJ female rats. TSS = transcription start site (ENSRNOTO0000077054.1). ENCODE regions of DNase I hypersensitivity and p300/CBP binding from mouse forebrain are also shown (green and gray boxes, respectively). Two genomic regions (site 1 and site 2, shaded blue) enriched for $\mathrm{H} 3 \mathrm{~K} 4 \mathrm{me} 1$ and $\mathrm{H} 3 \mathrm{~K} 27 \mathrm{ac}$ were identified as putative enhancers of the Kiss1 gene. b Abundance of H3K4me1 (dark green), H3K27ac (red), p300/CPB (red), MLL3 (light green), MLL4 (orange), MLL1 (yellow), EED (dark blue), and H3K27me3 (light blue) at enhancer sites 1 and 2 in the ARC throughout prepubertal development. Bars represent mean \pm s.e.m. $\left(n=6-8\right.$ per group). ${ }^{\star} P<0.05$ and ${ }^{\star \star} P<0.01$ vs. INF; one-way ANOVA followed by SNK test 
chromatin landscape of the promoter unchanged. The control constructs dSaCas9-KRAB (CK) and dSaCas9-sg4 lacking the $\mathrm{KRAB}$ domain $(\Delta \mathrm{K})$, and the construct dSaCas9-KRAB-sg1 were similarly ineffective in altering $\mathrm{H} 3 \mathrm{~K} 9 \mathrm{me} 3$ abundance (Fig. 7b), indicating that the effect of dSaCas9-KRAB-sg4 on H3K9me3 abundance at site 1 is specific and unrelated to a potential nonspecific effect of KRAB. The increase in $\mathrm{H} 3 \mathrm{~K} 9 \mathrm{me} 3$ abundance at site 1 was accompanied by decreased MLL3 occupancy, without a significant change in EED recruitment (Fig. 7d), suggesting that KRAB-sgRNA-mediated silencing diminishes MLL3 access to the enhancer, without facilitating PcG recruitment.

In contrast to $\mathrm{H} 3 \mathrm{~K} 9 \mathrm{me} 3$, basal levels of $\mathrm{H} 3 \mathrm{~K} 27 \mathrm{ac}$ were not affected at either site 1 or the Kiss 1 promoter (Fig. 7e, f). This result is consistent with an earlier report showing that dCas9KRAB does not alter H3K27ac content at another enhancer site ${ }^{34}$.
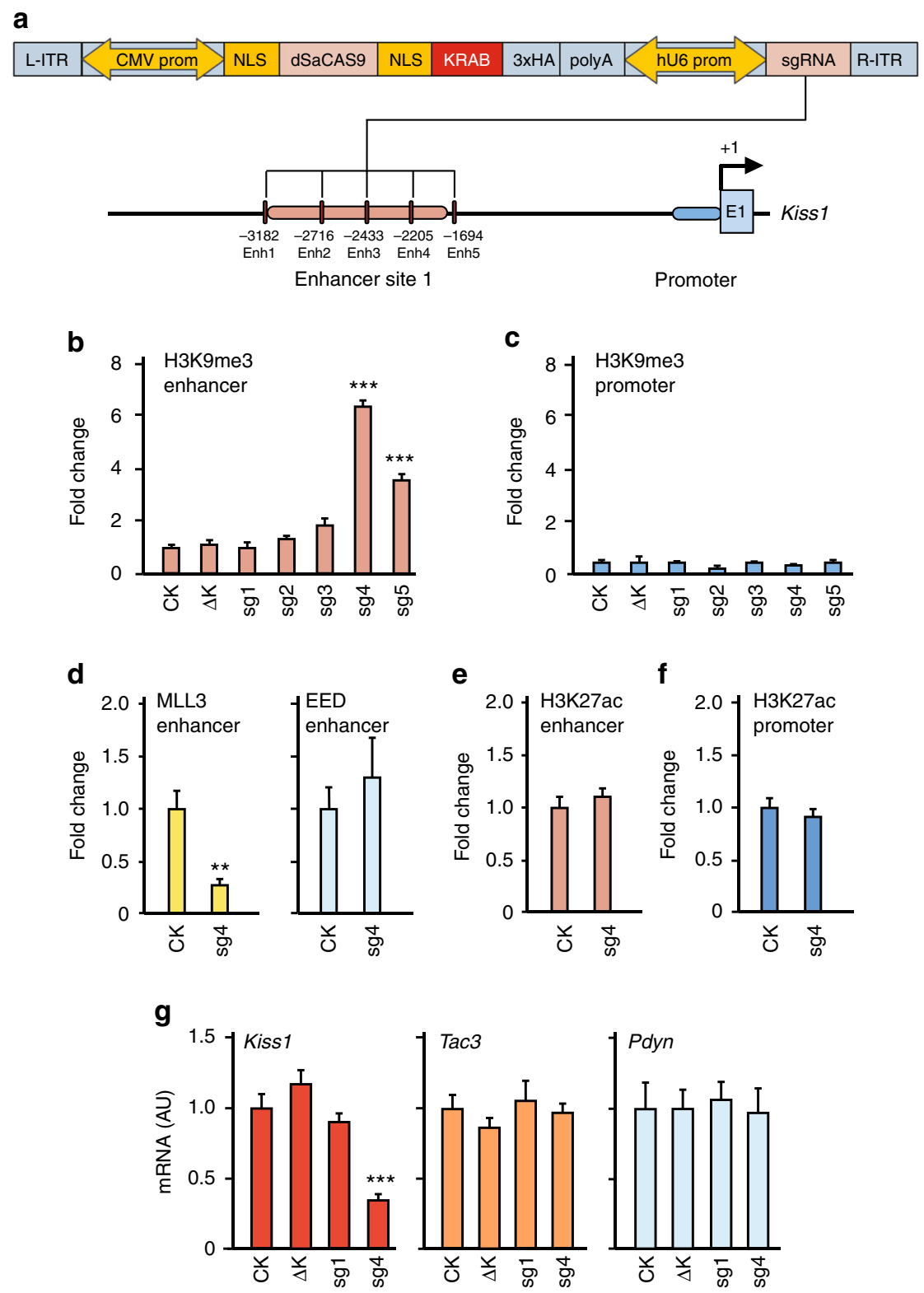

Fig. 7 CRISPR-dSaCas9-KRAB-mediated epigenome silencing of the ARC Kiss1 enhancer. a Schematic representation of the AAV-dSaCas9-KRAB construct used for epigenome editing of the Kiss1 enhancer. The AAV plasmid backbone contains a CMV promoter driving expression of dSaCas9-KRAB, two nuclear localization signals (NLS) flanking dSaCas9, and three copies of an influenza hemagglutinin (3xHA) tag. It also contains a single-guide (sg) RNA cloning site and a U6 promoter driving sgRNA expression. sgRNAs complementary to five different sequences in site 1, the putative Kiss1 enhancer domain, were identified using an online tool (https://www.deskgen.com/) and cloned into the AAV-dSaCas9-KRAB vector as recommended 66 . b H3K9me3 content at the Kiss1 enhancer (site 1) or c Kiss1 promoter as assessed by ChIP-qPCR 2 days after transfecting Rat1 cells with AAV-dSaCas9-KRAB-sgRNAs 1-5 (sg1-5) or either of two control constructs, AAV-dSaCas9-KRAB (CK) or AAV-dSaCas9 ( $\triangle \mathrm{K})(n=3$, experiment run in duplicate). d MLL3 and EED occupancy of the Kiss1 enhancer site 1 in cells transfected with CK or dSaCas9-KRAB-sgRNA4 (sg4) ( $n=3$, experiment run in duplicate). e, $\mathbf{f}$ H3K27ac content at the Kiss1 enhancer site 1 (e) and Kiss1 promoter (f) in CK and sg4 transfected cells ( $n=3$, experiment run in duplicate). g Kiss1, Tac3, and Pdyn mRNA expression in Rat1 cells transfected with either CK $(n=6), \Delta K(n=4)$, sg1 $(n=6)$, or sg4 $(n=8)$. RNA expression data were normalized by using peptidylprolyl isomerase $A$ as the housekeeping gene and dividing each individual value by the average of the CK group. ChIP-PCR data were also normalized by dividing each individual value by the average of the CK group. Bars represent mean \pm s.e.m. (in $\mathbf{b}, \mathbf{c}, \mathbf{g}$ : ${ }^{\star \star \star} P<0.001$ vs. control (CK) cells; one-way ANOVA followed by the Dunnett's test; in $\mathbf{d}-\mathbf{f}$ : ${ }^{\star \star} P<0.01$ vs. control (CK) cells; Student's $t$-test). AU = arbitrary units 
Considered together, these two observations suggest that a change in basal H3K27ac content is not a core feature of KRAB-mediated epigenomic silencing. Instead, H3K9 methylation appears to be the hallmark of KRAB-mediated gene repression ${ }^{37}$, and the main feature of dCas9-KRAB-induced epigenetic silencing at enhancer $\operatorname{sites}^{33,35}$. In keeping with this view, Kiss 1 mRNA levels were markedly reduced by sg4, without alterations in either Tac3 or $P d y n$ content (Fig. 7g). As was the case of H3K9me3 (Fig. 7b), AAV-dSaCas9-KRAB-sg1 (sg1) had no effect on Kiss1 mRNA levels, which were similar to levels detected in cells exposed to either $\mathrm{CK}$ or $\Delta \mathrm{K}$ (Fig. $7 \mathrm{~g}$ ). By demonstrating that epigenetic silencing of site 1 results in selective loss of Kiss 1 expression, these results identify site 1 as a bona fide ARC Kiss 1 enhancer.

The ARC Kiss1 enhancer is required for pubertal timing. To determine the physiological consequences of silencing the ARC Kiss1 enhancer in vivo we bilaterally targeted the ARC of EJ female rats with an AAV-dSaCas9-KRAB construct carrying sgRNA4 (sg4). Control (CK) animals were injected with the AAV-dSaCas9-KRAB construct devoid of sgRNAs (AAV-dSaCas9-KRAB, CK). We then followed the reproductive development of these animals to assess potential alterations in the time of puberty and reproductive cyclicity. Rats with correctly placed sg4 (determined by the elevated levels of Cas 9 mRNA in the ARC and a considerable lower expression in the lateral hypothalamus (Fig. 8a) had a markedly delayed age at VO (Fig. 8b; age at VO; C, $32.0 \pm 0.35$ days vs. $s g 4,36.3 \pm 0.90$ days; $t$ $=4.12, P<0.01$, Student's $t$-test), and an even more pronounced delayed age at first estrous (Fig. 8c, age at first estrous; C, $33.9 \pm$ 0.43 days vs. $s g 4,41.3 \pm 1.48$ days; $t=4.46, P<0.001$, Student's $t$ test). Because ovulation occurs on the day of the first estrous ${ }^{9}$, this result indicates that silencing the ARC Kiss1 enhancer severely postpones not only the initiation but also the completion of puberty. Whereas all control animals had VO by PND34, only $29 \%$ of sg 4 rats exhibited VO at this time (Fig. 8 b). Similarly, only $14 \%$ of the sg4-injected rats had ovulated at the age when all control rats had already ovulated (Fig. 8c). In addition to these alterations, estrous cyclicity was strikingly disrupted, with the sg4-injected animals showing many more days in an intermediate stage (estrous/diestrus, ED) than in proestrus or estrous than controls (Fig. 8d, e).

To determine if silencing the ARC Kiss1 enhancer in vivo resulted in a similar loss of Kiss 1 expression in the ARC as seen in a

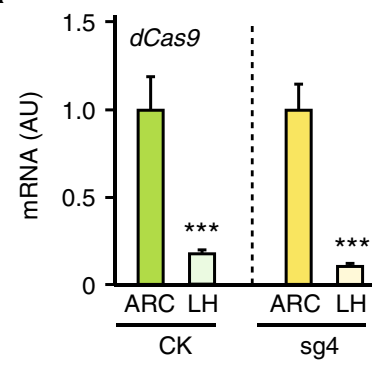

d

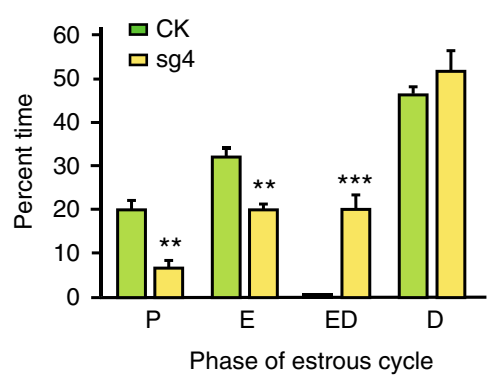

g
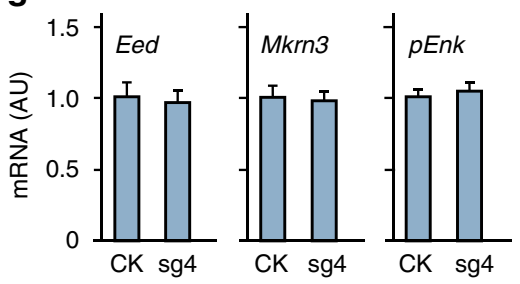

b

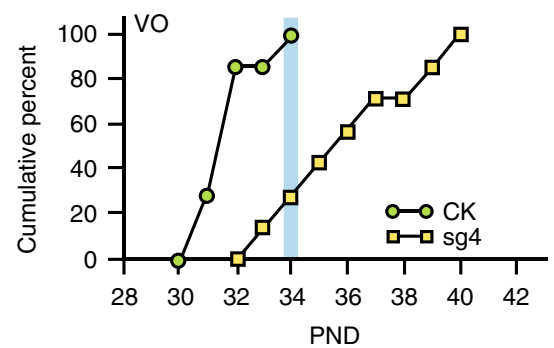

e

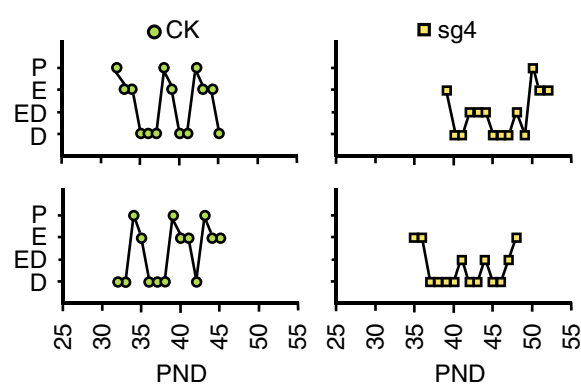

C

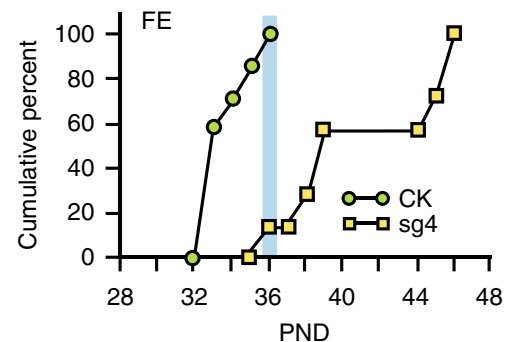

f

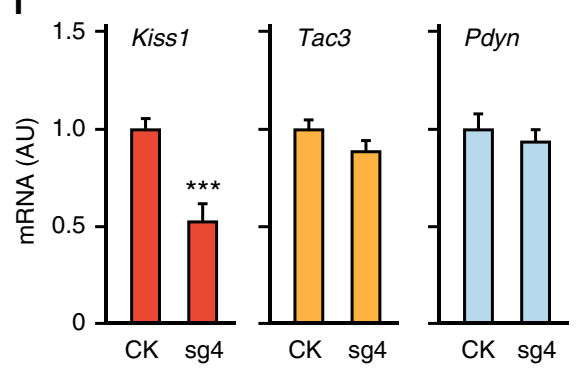

Fig. $8 \mathrm{KRAB}$-sgRNA-mediated epigenome editing of the ARC Kiss1 enhancer in the ARC of immature female rats. a dSaCas9 mRNA content in the ARC as compared with the lateral hypothalamus $(\mathrm{LH})$ in rats microinjected bilaterally with sg 4 or CK targeting the ARC. $\mathbf{b}$ Cumulative percent of animals showing vaginal opening (VO), and $\mathbf{c}$ first estrus (FE) in control CK or sg4-injected rats. Blue shade represents the time when all C animals had VO or FE. $\mathbf{d}$ Percent of time that rats injected with CK or sg4 spent in different stages of the estrous cycle. e Examples of estrous cycle patterns in rats injected with CK or sg4. $\mathbf{f}$ Kiss1, Tac3, and Pdyn mRNA levels in the ARC of animals injected with CK or sg4 detected by qPCR. $\mathbf{g}$ Expression of puberty-inhibitory genes (blue bars) or puberty-activating genes (magenta bars) in the ARC of female rats receiving CK or sg4. RNA expression data were normalized by using peptidylprolyl isomerase $A$ as the housekeeping gene and dividing each individual value by the average of the $C K$ group. $P=$ proestrous; $E=e s t r o u s ; E D=$ transitional phase (estrous/diestrous); $\mathrm{D}=$ diestrous; $\mathrm{AU}=$ arbitrary units ( $n=7-8$ per group). Bars represent mean \pm s.e.m. ( ${ }^{\star \star} P<0.01,{ }^{\star \star \star} P<0.001$ vs. $C K$-injected controls, Student's t-test) 
cells in vitro, we collected the ARC of all animals at the diestrous phase and after all controls had undergone three complete estrous cycles, and measured Kiss 1 , Tac3, and Pdyn mRNA content, in addition to several other mRNAs (see below). In agreement with the in vitro results, Kiss1 mRNA abundance was reduced in animals injected with sg4 as compared to controls (Fig. 8f). In contrast, neither Tac3 nor Pdyn mRNA levels) were affected (Fig. 8f), as was the case for 11 other genes involved in the inhibitory (Eed, Mkrn3, and $p E n k$ ) and stimulatory (Ttf1, Nell2, Eap1, Gpr54, Tac3R, Grin 2a, Grin2d, and Grik5) control of puberty (Fig. 8g). Thus, in vivo epigenome silencing of the ARC Kiss1 enhancer results in selective loss of Kiss 1 expression in the ARC, delays the initiation and normal progression of the pubertal process, and significantly compromises the normalcy of reproductive cyclicity.

\section{Discussion}

Our results suggest that the timely initiation of female puberty requires a dual, coordinated action of COMPASS-like proteins exerted on Kiss 1 and Tac3, two genes that-expressed in KNDy neurons of the hypothalamus-are essential for both puberty to occur and reproductive function to proceed ${ }^{6,7,38}$. On the one hand, MLL1 recruited to the promoter region of these two genes establishes a permissive chromatin configuration required for transcriptional activation. On the other hand, MLL3 acts at an ARC-specific Kiss1 enhancer site to implement a chromatin structure that facilitates enhancer activation. As these changes take place, eviction of EED from the Kiss1 enhancer and from the Kiss1 and Tac3 promoters results in loss of PcG-mediated repression ${ }^{9}$ at each of these genomic sites.

The earlier increase in $\mathrm{H} 3 \mathrm{~K} 4 \mathrm{me} 2$ than in $\mathrm{H} 3 \mathrm{~K} 4 \mathrm{me} 3$ abundance observed at both the Kiss 1 and Tac3 promoters before puberty appears to be particularly relevant to the pubertal activation of Kiss1 and Tac3 expression. Methylation of lysine 4 at $\mathrm{H} 3$ follows an orderly sequence with monomethylation taking place first, followed by di- and trimethylation thereafter ${ }^{20}$. Additionally, trimethylation requires a longer interaction of the methylating enzyme with its unmethylated H3K substrate ${ }^{14}$. It is, therefore, likely that the earlier increase in $\mathrm{H} 3 \mathrm{~K} 4 \mathrm{~m} 2$ abundance at the Kiss 1 and Tac3 promoter simply reflect the developmental unfolding of this biosynthetic sequence. It also implies that $\mathrm{H} 3 \mathrm{~K} 4 \mathrm{me} 2$ facilitates the initial prepubertal activation of Kiss 1 and Tac3 expression, which is then further enhanced and sustained by $\mathrm{H} 3 \mathrm{~K} 4 \mathrm{me} 3$. Indeed, $\mathrm{H} 3 \mathrm{~K} 4 \mathrm{me} 2$ is abundant at regulatory regions (such as promoters) targeted by transcription factors ${ }^{39}$, and at poised (i.e., ready to be activated) promoters ${ }^{40}$, such as Kiss 1 and Tac3 before puberty. H3K4me3 abundance, on the other hand, is higher at the promoter of active genes ${ }^{40}$. Our results are consistent with these concepts as they show elevated levels of H3K4me2 at both the Kiss 1 and Tac3 promoters preceding (Kiss1) or accompanying (Tac3) the prepubertal increase in expression of these genes, whereas H3K4me3 levels become elevated at the juvenile-pubertal transition (LJ period), coinciding with enhanced Kiss 1 and Tac3 expression.

The critical importance that COMPASS-like proteins have for the coordinated regulation of these two puberty-activating genes, and hence for the pubertal process itself, is demonstrated by the striking delay of puberty and loss of reproductive cyclicity resulting from either ARC-specific RNAi-mediated knockdown of Mll1 expression or epigenome silencing of the MLL3-supported ARC Kiss1 enhancer. While RNAi-mediated loss of hypothalamic Mll1 expression led to a reduction in both Kiss 1 and Tac3 expression, CRISPR-dCas9-KRAB-sgRNA4-mediated epigenome silencing of the ARC Kiss1 enhancer, resulted in selective loss of Kiss1 expression. Yet, in both cases the net in vivo outcome was similar, as the advent and completion of puberty were delayed and reproductive cyclicity was disrupted. This similarity would be expected because Kiss 1 and Tac3 operate along a common biological pathway in KNDy neurons.

The estrous cycle of both LV-sh2612 and AAV-dSaCas9KRAB-sg4 (sg4)-injected animals showed a predominance of an intermediate stage between estrous and diestrus, suggesting that the basic recurrent pattern of pulsatile and surge LH release necessary for normal reproductive cyclicity was impaired. This long-term alteration of estrous cyclicity may be due to maintained loss of MLL1 in the former case, and persistent inhibition of enhancer activity by continuous replenishment of the heterochromatin complex drawn by the KRAB domain ${ }^{37}$ in the latter. Although epigenome silencing of the ARC Kiss1 enhancer appeared to be more effective than ARC loss of Mll1 in both delaying puberty and establishing a deranged pattern of estrous cyclicity, Kiss 1 mRNA levels in the ARC were similar in both groups implying that the seemingly different biological outcome of these manipulations may just reflect physiological variability in the response of animals treated in different experiments.

We demonstrate that CRISPR-dCas9-KRAB-mediated epigenome silencing of a distal enhancer alters a specific developmental milestone, such as puberty, in an in vivo setting. In agreement with previous in vitro result ${ }^{33}$, our findings also show that sg4-mediated epigenome editing increases the abundance of the repressive histone mark $\mathrm{H} 3 \mathrm{~K} 9 \mathrm{me} 3$ only at the enhancer site, without affecting the content of this mark at the Kiss 1 promoter. In contrast to $\mathrm{H} 3 \mathrm{~K} 9 \mathrm{me} 3$, the abundance of $\mathrm{H} 3 \mathrm{~K} 27 \mathrm{ac}$ at the Kiss 1 enhancer remained unaltered, a result consistent with an earlier finding showing that dCas9-KRAB does not reduce the H3K27ac content of another enhancer ${ }^{34}$. While loss of H3K27ac may not be a core feature of KRAB-mediated epigenomic silencing, H3K9 methylation has been shown to be a hallmark of KRAB-mediated gene repression ${ }^{37}$, and the main feature of dCas9-KRAB-induced epigenetic silencing at enhancer sites ${ }^{33,35}$. Because the increase in H3K9me3 abundance at site 1 was accompanied by loss of MLL3 occupancy without increased EED recruitment, it can be concluded that exclusion of MLL3 from the Kiss1 enhancer contributes to KRAB-mediated epigenome silencing, but that the repressive complex recruited by the KRAB-sgRNA platform does not involve increased EED occupancy. The above described changes were accompanied by decreased Kiss 1 expression in the absence of significant alterations in the expression of a host of other genes involved in the stimulatory and inhibitory control of puberty, indicating that dCas9-KRAB-sgRNA-mediated epigenome silencing targeting the Kiss1 enhancer is a site-specific phenomenon. Consequently, our results provide a significant step forward toward defining the functional impact that modification of an enhancer has on a physiological function at an organismal level, a sought-after goal in genomic biology ${ }^{29,41}$. At a developmental neuroendocrine level, our results indicate that the timely activation of the ARC Kiss 1 enhancer is critical for both setting in motion the pubertal process and guiding it to completion.

Another interesting outcome of this study is the specificity of the MLL1 stimulatory effects on the two main puberty-activating genes expressed in KNDy neurons. MLL1 was recruited to the promoter region of only Kiss 1 and Tac3, but not to the promoter of the restraining gene Pdyn. In vitro experiments showed that in addition to the Kiss 1 and Tac3 promoters, MLL1 is able to transactivate the promoters of three other puberty-activating genes (Ttf1, Nll2, and Eap1). However, a transcriptome analysis of these and 10 additional genes involved in the stimulatory and inhibitory control of puberty, demonstrated that in no instance expression of these genes was reduced in the ARC of LV-sh2612injected animals. A plausible explanation for this apparent discrepancy was provided by ChIP assays showing that in contrast to 
the puberty-related increase in MLL1 association to the Kiss1 and Tac3 promoters, MLL1 recruitment to the Ttf1 and Nell2 promoters (selected as examples of puberty-activating genes) does not increase at puberty. TTF1 is a transcription factor involved in the stimulatory control of Kiss1 transcription ${ }^{23}$ and NELL2 is a protein selectively expressed in glutamatergic neurons that also contribute to facilitating female puberty ${ }^{24}$. These results suggest that although MLL1 is able to stimulate the transcription of genes other than Kiss 1 and Tac3 under defined in vitro conditions, this effect might not occur at puberty, because recruitment of MLL1 to the promoters of these genes does not increase at this time.

Based on these considerations it can be concluded that an important function of MLL1 and MLL3 in the prepubertal hypothalamus is to steer the epigenetic balance controlling reproductive development via Kiss1 and Tac3 away from a repressive, PcG-mediated mode toward a predominantly stimulatory TrxG-driven status. By implementing histone modifications associated with transcriptional activation at specific genomic loci involved in the stimulatory control of puberty, the TrxG complex provides the necessary trans-activational drive to puberty-activating genes at the time when the strength of PcGmediated epigenetic silencing is waning. The potential relevance of TrxG/PcG-dependent epigenetic regulation in the control of human puberty is suggested by the finding that inactivating mutations of $\mathrm{CHD}^{42,43}$, a TrxG-related chromatin-remodeling protein that antagonizes PcG action, causes hypothalamic hypogonadism.

Altogether, the present results demonstrate a role of a PcG/ TrxG-counterbalancing mechanism in the process by which the neuroendocrine brain controls the initiation of puberty, thereby broadening significantly the scope of developmental events controlled by PcG and TrxG proteins. In addition to being required for specification of early developmental processes ${ }^{12}$, our results suggest that PcG/TrxG-dependent balance of gene expression is a core component of one of the most fateful events of postnatal mammalian development, the acquisition of reproductive competence.

\section{Methods}

Animals. We used Sprague Dawley female rats (Charles River Laboratories International, Inc. (Hollister, CA) at four different phases of postnatal development: INF (PND14); EJ (PND21); LJ (PND28); and PUB (PND29-35). The Oregon National Primate Research Center (ONPRC) Animal Care and Use Committee approved the use of these animals in accordance with established NIH guidelines. The animals were obtained from, randomly assigned to different experimental groups, and housed in a room with controlled photoperiod (12/12 h light/dark cycle) and temperature $\left(23-25^{\circ} \mathrm{C}\right)$, with ad libitum access to tap water and pelleted rat chow.

Hypothalamic tissue from female rhesus monkeys (Macaca mulatta) was obtained through the ONPRC Tissue Distribution Program. Animals were classified into different stages of pubertal development based on the age of the animals and the pubertal stages reported by Watanabe and Terasawa ${ }^{44}$.

Evaluation of sexual maturation and estrous cyclicity. We measured changes in hypothalamic gene expression and chromatin composition at three phases of female rat prepubertal development, INF, EJ, and LJ. At PND21 animals are in the EJ phase ${ }^{45}$. The vagina is closed and the uterus weighs $60 \mathrm{mg}$ or less, and is devoid of intrauterine fluid. At PND28, the rats are considered to be in the LJ phase; the vagina remains closed and the uterus lacks fluid. $L J$ animals display a diurnal change in pulsatile plasma LH levels, with the amplitude of LH pulses increasing in the afternoons ${ }^{46}$. In rats, as well as in humans and nonhuman primates, this change signals the increase in hypothalamic drive that initiates puberty ${ }^{47}$.

To determine the effect of intrahypothalamic injections of a LV construct (LVshMll1-GFP) targeting a shRNA to Mll1 mRNA (sh2612) or an AAV vector (AAVdSaCas9-sgRNA4, sg4) targeting the ARC-specific Kiss1 enhancer on the onset of puberty and subsequent estrous cyclicity. Starting 5 days after the injections, the animals were inspected every morning for VO. Thereafter, we performed daily vaginal lavages to assess the occurrence of the first estrous, a stage in which most cells in the lavage are cornified. Vaginal cornification followed by a predominance of leukocytes was considered as proof that ovulation had occurred. An abundance of leukocytes defines the diestrous phase of the estrous cycle and indicates that a functional corpus luteum was formed after ovulation. Accordingly, the age at first ovulation was considered to have occurred only when the cornified cells were replaced by at least 2 days of vaginal lavages displaying mostly leukocytes 9 . In all cases, estrous cyclicity was monitored for at least 14 days after VO, a period during which control animals had experience at least four estrous cycles.

Induction of a preovulatory surge of estrogen. To induce a preovulatory surge of plasma estradiol levels that sets in motion the LP phase of puberty, we injected 26day-old female rats intraperitoneally (i.p.) with $5 \mathrm{IU}$ of pregnant mare serum gonadotropin (Sigma-Aldrich, St. Louis, MO, USA), as previously described ${ }^{48}$. The $\mathrm{MBH}$ from each animal was collected $48 \mathrm{~h}$ later for molecular analysis.

Tissue collection. The MBH of female rats was dissected by four cuts, one rostral adjacent to the posterior border of the optic chiasm, one caudal cut along the anterior edge of the mammillary bodies, and two lateral cuts placed between the medial eminence and the hypothalamic sulci. The fragments obtained had a thickness of about $2 \mathrm{~mm}$. This fragment includes the entire ARC. The lateral hypothalamus collected in one experiment consisted of tissue dissected by lateral cuts made along the hypothalamic sulci and each of the two cuts previously made half-way between the hypothalamic sulci and the median eminence. A fragment of the CTX was also collected. Upon dissection, the tissues were immediately frozen on dry ice and stored at $-85^{\circ} \mathrm{C}$ until RNA or chromatin extraction. The MBH of female monkeys was dissected as described earlier ${ }^{25}$.

Stereotaxic delivery of viral particles. We used both LV and AAV viral constructs (for description of these constructs see corresponding sections below). We delivered LV particles carrying an shRNA directed against Mll1 mRNA (termed sh2612) or a control lentivirus expressing GFP (C) to the ARC of 21-day-old EJ female rats. Other animals received an AAV construct encoding a nucleasedeficient Cas9 (dCas9) fused with a KRAB repressor domain that is targeted to the ARC Kiss1 enhancer sequence by a sgRNA (sg4). Control animals were injected with an AAV devoid of sgRNAs (CK). The animals were anesthetized with a cocktail of ketamine $\left(25 \mathrm{mg} \mathrm{ml}^{-1}\right)$, xylazine $\left(5 \mathrm{mg} \mathrm{m}^{-1}\right)$, and acepromazine $(1 \mathrm{mg}$ $\left.\mathrm{ml}^{-1}\right)$, administered i.p. $(0.15 \mathrm{ml}$ per $100 \mathrm{~g}$ of body weight). Thereafter, the rats were positioned on a stereotaxic instrument (David Kopf Instruments, Tujunga, CA) with the incisor bar set at $+5 \mathrm{~mm}$. A total volume of $1 \mu \mathrm{l}$ containing control viruses (C or $\mathrm{CK}$ ), sh2612, or sg4 was injected bilaterally into the ARC at the rate of $250 \mathrm{nl} \mathrm{min}^{-1}$, using a $10 \mu \mathrm{l}$ Hamilton micro-syringe connected to a Stoelting Stereotaxic microinjector (Stoelting, Wood Dale, IL). The coordinates used were as follows: $0.3 \mathrm{~mm}$ lateral from midline; $0.2 \mathrm{~mm}$ anterior from bregma; and $9.6 \mathrm{~mm}$ vertical from the surface of the skull ${ }^{25}$. The surgical procedure lasted about $15 \mathrm{~min}$. Following surgery, the animals were placed in a clean cage on a heating pad unti returned to their home cages. Thereafter, they were treated for 3 days with an analgesic (Carprofen, $5 \mathrm{mg} \mathrm{kg}^{-1}$ ) and an antibiotic (Baytril $10 \mathrm{mg} \mathrm{kg}^{-1}$ ), administered subcutaneously.

RNA extraction, reverse transcription, and QPCR. Total RNA was extracted from tissues (MBH and CTX) and cultured cells using the RNeasy mini kit (Qiagen, Valencia, CA) following the manufacturer's instructions. RNA concentrations were determined by spectrophotometric trace (Nanodrop, ThermoScientific, Wilmington, DE). Total RNA (500 ng) was transcribed into cDNA in a volume of $20 \mu \mathrm{l}$ using $4 \mathrm{U}$ Omniscript reverse transcriptase (Qiagen). We measured the mRNAs of interest using the SYBR GreenER ${ }^{\mathrm{TM}}$ qPCR SuperMix system (Invitrogen, Carlsbad, CA), and primers (Supplementary Table 1) designed with help of either DNASTAR 14 software (PrimerSelect tool, Madison, WI) or the Primer-Blast program from NCBI. All PCR reactions were carried out using a QuantStudio 12K Real-Time PCR system in a $10 \mu \mathrm{l}$ volume, with $1 \mu \mathrm{l}$ of cDNA or a reference cDNA (for details see below), $4 \mu \mathrm{l}$ of primers (each primer at $1 \mu \mathrm{M}$ each), and $5 \mu \mathrm{l}$ of SYBR GreenER ${ }^{\mathrm{TM}} \mathrm{qPCR}$ SuperMix. The PCR reaction was performed using the following conditions: $95^{\circ} \mathrm{C}$ for $5 \mathrm{~min}$, followed by 40 cycles of $15 \mathrm{~s}$ at $95^{\circ}$ $\mathrm{C}$ and $60 \mathrm{~s}$ at $60^{\circ} \mathrm{C}$. Formation of a single SYBR Green-labeled PCR amplicon was verified by running a three-step melting curve analysis after each reaction $(15 \mathrm{~s}$ at $95^{\circ} \mathrm{C}, 1 \mathrm{~min}$ at $60^{\circ} \mathrm{C}$, increasing to $95^{\circ} \mathrm{C}$ at $0.5^{\circ} \mathrm{C} \mathrm{s}^{-1}$, with detection every $0.5 \mathrm{~s}$, and ending at $95^{\circ} \mathrm{C}$ for $15 \mathrm{~s}$ ).

Threshold cycles (CTs) for each PCR reaction were identified using by QuantStudio 12K Flex software. To construct relative standard curves, we used serial dilutions $(1 / 2$ to $1 / 500)$ of a pool of $c D N A s$ generated by mixing equal amounts of cDNA from each sample. The CTs from each sample were referred to the relative standard curve to estimate the mRNA content per sample; the values obtained were normalized for procedural losses using glyceraldehyde-3-phosphate dehydrogenase $(G A P D H)$ mRNA or peptidylprolyl isomerase A (Ppia) as the normalizing unit.

Massively parallel RNA sequencing. Total RNA from the MBH of female rats at different stages of prepubertal development was subjected to RNA-seq. The RNAseq procedure was carried out by the OHSU Massively Parallel Sequencing Shared Resource. RNA-seq libraries were prepared using the TruSeq Stranded protocol with ribosomal reduction (Illumina, San Diego, CA). Briefly, $600 \mathrm{ng}$ of total RNA per sample were depleted of ribosomal RNA using RiboZero capture probes 
(Illumina). The purified RNA was then fragmented using divalent cations and heat, and the fragmented RNA was used as template for reverse transcription using random hexamer primers. The resulting cDNAs were enzymatically treated to blunt the ends, and a single "A" nucleotide was added to the $3^{\prime}$-ends to facilitate adaptor ligation. Standard six-base pair Illumina adaptors were ligated to the cDNAs and the resulting DNA was amplified by 12 rounds of PCR. All of the above procedures were carried out following the protocol provided by Illumina. Unincorporated material was removed using AMPure XP beads (BeckmanCoulter, Brea, CA). Libraries were profiled on a Bioanalyzer instrument (Agilent, Santa Clara, CA) to verify: (a) the distribution of DNA sizes in the library; and (b) the absence of adapter dimers. Library titers were determined using real-time PCR (Kapa Biosystems, Wilmington, MA) on a StepOnePlus Real Time System (ThermoFisher, Waltham, MA). Libraries were mixed to run four samples per lane on the HiSeq 2500 (Illumina). Sequencing was done using a single-read 100-cycle protocol. The resulting base call files (.bcl) were converted to standard fastq formatted sequence files using Bcl2Fastq (Illumina). Sequencing quality was assessed using FastQC (Babraham Bioinformatics, Cambridge, UK).

RNA-seq data analysis. To determine the differential expression of genes during pubertal development we used the gene-level edge $\mathrm{R}^{49}$ analysis package. We performed an initial trimming and adapter removal pass using Trimmomatic ${ }^{50}$. Reads that passed Trimmomatic processing were aligned to the rn6 build of the rat genome with Bowtie2/Tophat $2^{51,52}$, and assigned to gene-level genomic features with the Rsubread featureCounts package based on the Ensembl 83 annotation set. Differential expression between time points was analyzed using the generalized linear modeling approaches implemented in edgeR. Batch effect terms were included in these models to correct for runs on different dates/flow cells. Lists of differentially expressed genes/transcripts were identified based on significance of pairwise comparison of time points to identify the genes most likely to be differentially expressed for later reverse transcription (RT)-qPCR confirmation.

Functional promoter assays. To determine if MLL1 alters the transcription of several putative downstream genes (KISS1, TAC3, Ttf1, PDYN, Eed, Eap1, PENK, Nell2, and MKRN3) we used Neuro2A cells (N2A, American Type Culture Collection (ATCC), Manassas, VA) transfected with luciferase reporter constructs containing the TSS and adjacent sequence of these genes (Supplementary Table 2) PCR-cloned into the SmaI/SacI sites of pGL2 (Promega, Madison, WI), in addition to the expression vectors Mll1-pcXN2 (kindly provided by Thomas Milne, University of Oxford, Headington, Oxford, UK), Eed-pcDNA3.1, or a combination of both. The cells were cultured at $37^{\circ} \mathrm{C}$ in a humidified atmosphere containing $5 \%$ $\mathrm{CO}_{2}$. They were maintained in Dulbecco's modified Eagles medium (DMEM) containing high glucose $\left(4.5 \mathrm{gl}^{-1}\right.$; Sigma), supplemented with $10 \%$ fetal bovine serum (FBS; Invitrogen), L-glutamine $\left(2.5 \mathrm{mM}\right.$, Sigma), $100 \mathrm{U} \mathrm{ml}^{-1}$ penicillin, and $100 \mathrm{\mu g} \mathrm{ml}^{-1}$ streptomycin (Invitrogen). For the assays, the cells (400,000 cells per well) were seeded onto 24-well plates in DMEM containing $10 \% \mathrm{FBS}$. After $24 \mathrm{~h}$, we transiently co-transfected the reporter constructs (using as a backbone the luciferase reporter plasmid pGL2) along with the pcXN2-Mll1 construct and Lipofectamine 2000 (Invitrogen) at a ratio of $1 \mu \mathrm{g}$ DNA:2.5 $\mu$ l Lipofectamine 2000 in Optimem (Invitrogen). After $5 \mathrm{~h}$ of transfection, the cells were returned to serum-containing DMEM medium; $48 \mathrm{~h}$ later, they were harvested and lysed in $150 \mu \mathrm{l}$ lysis buffer from the Firefly Luciferase Glow Assay Kit (Pierce, Rockford, IL). Cell lysate were spun at $10,000 \times g$ for $10 \mathrm{~min}$ and $100 \mu \mathrm{l}$ supernatant was assayed for luciferase activity using $70 \mu \mathrm{l}$ Luciferase reagent. All assays were carried out in opaque 96-well plates, and a Spectramax M5 microplate reader (Molecular Devices, Sunnyvale, CA) was employed to measure light emission. To assess transfection efficiency we co-transfected the plasmid CMV-Sport- $\beta$-gal (Invitrogen) at $10 \mathrm{ng} \mathrm{ml}$ ${ }^{-1}$, and determined $\beta$-galactosidase activity using $10 \mu \mathrm{l}$ cell lysate and $100 \mu \mathrm{l}$ Tropix Galacto Light Plus (ABI) reagent. $\beta$-Galactosidase activity was also determined in opaque 96-well plates and light emission was measured as indicated above.

ChIP assay. To assess the recruitment of TrxG proteins to specific gene promoters, and the association of different histone modifications to either these promoters or putative distal enhancer domains in vivo, we performed ChIP assays using chromatin extracted from the $\mathrm{MBH}$ of prepubertal female rats (PND14, 21, and 28). In some cases, chromatin was also extracted from the CTX. To assess the changes in histone modifications resulting from expression of dSaCas9-KRAB-sgRNA constructs, ChIP assays were performed using chromatin extracted from Ratl cells transfected with these constructs. The ChIP procedure was described previously by us $^{9,23}$, and was carried out with minimal modifications. Cells were harvested for ChIP $48 \mathrm{~h}$ after transfection. The cells and tissue fragments were washed once in ice-cold phosphate-buffered saline (PBS) containing a protease inhibitor cocktail (PI, $1 \mathrm{mM}$ phenylmethylsulfonylfluoride, $7 \mu \mathrm{g} \mathrm{ml}^{-1}$ aprotinin, $0.7 \mu \mathrm{g} \mathrm{m} l^{-1}$ pepstatin $\mathrm{A}$, and $0.5 \mu \mathrm{g} \mathrm{m}^{-1}$ leupeptin), a phosphatase inhibitor cocktail (PhI, $1 \mathrm{mM} \beta$ glycerophosphate, $1 \mathrm{mM}$ sodium pyrophosphate, and $1 \mathrm{mM}$ sodium fluoride), and an HDAC inhibitor (20 mM sodium butyrate). Thereafter, cells and tissue fragments were crosslinked by exposing them to $1 \%$ formaldehyde for $10 \mathrm{~min}$ at room temperature. After two additional washing steps in PBS the samples were lysed with $200 \mu \mathrm{l}$ SDS buffer $(0.5 \%$ SDS, $50 \mathrm{mM}$ Tris- $\mathrm{HCl}$, and $10 \mathrm{mM}$ EDTA) containing protease, phosphatase, and HDAC inhibitors, and sonicated for $45 \mathrm{~s}$ to yield chromatin fragments of $\sim 500$ base pairs (bp) using the microtip of a Fisher Scientific FB 705 sonicator. Size fragmentation was confirmed by agarose gel electrophoresis. The sonicated chromatin was clarified by centrifugation at 14000 r.p $\mathrm{m}$. for $10 \mathrm{~min}$ at $4{ }^{\circ} \mathrm{C}$, brought up to $1 \mathrm{ml}$ in Chip Dilution Buffer $(16.7 \mathrm{mM}$ Tris$\mathrm{HCl}, \mathrm{pH} 8.1,150 \mathrm{mM} \mathrm{NaCl}, 1.2 \mathrm{mM}$ EDTA, 1.1\% Triton X-100, and $0.01 \%$ SDS) containing the $\mathrm{PI}$ and $\mathrm{PhI}$ cocktails, and the HDAC inhibitor described above. The samples were then stored at $-80^{\circ} \mathrm{C}$ for subsequent immunoprecipitation. For this step, chromatin was pre-cleared with Protein A/G beads (Dynabeads, Invitrogen) for $1 \mathrm{~h}$ at $4{ }^{\circ} \mathrm{C}$. Twenty-five to $50 \mu \mathrm{l}$ aliquots of chromatin were then incubated with $2-5 \mu \mathrm{g}$ of the antibodies described in Supplementary Table 3 . The complexes were incubated with $25 \mu \mathrm{l}$ of protein $\mathrm{A}$ or $\mathrm{G}$ beads solution (Dynabeads) at $4{ }^{\circ} \mathrm{C}$ overnight with mild agitation. The next day the Ibeads were washed first with $0.5 \mathrm{ml}$ low-salt wash buffer (20 mM Tris-HCl, pH 8.1, $150 \mathrm{mM} \mathrm{NaCl}, 2 \mathrm{mM}$ EDTA, $1 \%$ Triton X-100, and $0.1 \%$ SDS), followed by high-salt wash buffer ( $20 \mathrm{mM} \mathrm{Tris-HCl,}$ $\mathrm{pH}$ 8.1, $500 \mathrm{mM} \mathrm{NaCl}, 2 \mathrm{mM}$ EDTA, $1 \%$ Triton X-100, and $0.1 \%$ SDS), LiCl buffer (10 mM Tris-HCl, pH 8.1, $250 \mathrm{M} \mathrm{LiCl}, 1 \%$ Nonidet P-40, 1\% sodium deoxycholate, and $1 \mathrm{mM}$ EDTA), and finally with TE buffer (10 mM Tris- $\mathrm{HCl}, \mathrm{pH} 8.0$, and $1 \mathrm{mM}$ EDTA). Thereafter, the immunocomplexes were eluted with $100 \mu \mathrm{l}$ of $0.1 \mathrm{M}$ $\mathrm{NaHCO}_{3}$ and $1 \%$ SDS at $65^{\circ} \mathrm{C}$ for $45 \mathrm{~min}$. To reverse the crosslinking reaction we added $4 \mu \mathrm{l}$ of $5 \mathrm{M} \mathrm{NaCl}$ and incubated the samples at $95^{\circ} \mathrm{C}$ for $30 \mathrm{~min}$. We recovered the DNA using ChIP DNA Clean \& Concentrator columns (Zymo Research, Irvine, CA), and stored the resulting material at $-80^{\circ} \mathrm{C}$ before $\mathrm{qPCR}$ analysis. All the chemicals mentioned above were purchased from Sigma-Aldrich.

qPCR detection of chromatin immunoprecipitated DNA. Genomic regions of interest were amplified by qPCR. Accession numbers of the genes analyzed as well as the chromosomal position of the $5^{\prime}$-flanking region amplified, using the position of the TSS as the reference point, are shown in Supplementary Table 1. The primer sequences (Eurofins MWG Operon, Huntsville, AL) used to detect the DNA fragment of interest in the immunoprecipitated DNA are also shown in Supplementary Table 1. PCR reactions were performed using $1 \mu \mathrm{l}$ of each immunoprecipitate (IP) or input samples (see below), primer mix (1 $\mu \mathrm{M}$ each primer), and SYBR Green Power Up Master Mix ${ }^{\mathrm{TM}}$ (Thermo Fisher) in a final volume of $10 \mu \mathrm{l}$. Input samples consisted of $10 \%$ of the chromatin volume used for immunoprecipitation. The thermocycling conditions used were as follows: $95^{\circ} \mathrm{C}$ for $5 \mathrm{~min}$, followed by 40 cycles of $15 \mathrm{~s}$ at $95^{\circ} \mathrm{C}$ and $60 \mathrm{~s}$ at $60^{\circ} \mathrm{C}$. Data are expressed as $\%$ of IP signal/input signal.

Genome-wide ChIP assays. To identify putative enhancer sites in the rat genome, ChIP assays were performed to assess the genome-wide distribution of H3K27Ac and $\mathrm{H} 3 \mathrm{~K} 4 \mathrm{mel}$ using chromatin extracted from the MBH of 28-day-old LJ female rats. The immunoprecipitated DNA was amplified before library preparation and deep-sequencing. Shortly, $10 \mu \mathrm{l}$ of immunoprecipitated DNA were dephosphorylated using Shrimp Alkaline Phosphatase (NEB, $1 \mathrm{U}$ ) for $10 \mathrm{~min}$ at $37^{\circ} \mathrm{C}$. Thereafter, thymidine residues were added to the DNA ends using Terminal Transferase (NEB, $20 \mathrm{U})$, in a T-tailing reaction $\left(20 \mathrm{~min}\right.$ at $\left.37^{\circ} \mathrm{C}\right)$ that utilized 5 $\mu \mathrm{M}$ dTTP and $5 \mu \mathrm{M}$ ddCTP. Upon completion of this reaction, a oligodeoxynucleotide adapter [T7-BpmI-oligo(A) 15 (5'-AATTAATACGACTCACTATAGGGCTGGAGAAAAAAAAAAAAAAA- ${ }^{\prime} ; 0.2 \mu \mathrm{M}$; Operon) was annealed to the ends of the DNA fragments and extended using $0.2 \mathrm{mM}$ dNTPs and Klenow fragment polymerase (NEB, $5 \mathrm{U}$ ) for $1 \mathrm{~h}$ at $37^{\circ} \mathrm{C}$. RNA was then synthesized by in vitro transcription using T7 RNA polymerase and the RNAMaxx high yield kit (Agilent) in an overnight reaction at $37^{\circ} \mathrm{C}$. The resulting RNA was purified using the RNeasy Mini Kit (Qiagen) and eluted in $20 \mu$ sterile water. Complementary DNA was then prepared using the T7-BpmI-oligo(A) 15 and the Superscript III reverse transcription Kit (Invitrogen), followed by second-strand synthesis using a 1:5 ratio TaqPolymerase (Roche, Branford, CT) and Pfu Polymerase (Agilent) in Thermopol Buffer (NEB) in a $200 \mu \mathrm{l}$ reaction for $30 \mathrm{~min}$ at $72^{\circ} \mathrm{C}$. The doublestranded DNA was purified using the QiaQuick PCR purification Kit (Qiagen) and eluted in $50 \mu \mathrm{l}$ sterile water. The purified DNA was then digested with BpmI (NEB) in a $60 \mu \mathrm{l}$ reaction for $2 \mathrm{~h}$ at $37^{\circ} \mathrm{C}$, followed by isolation using Zymo ChIP columns (Zymo Resaerch, Irvine, CA) and elution in $20 \mu \mathrm{l}$ sterile water. Eluted DNA was quantitated using the Qubit High Sensitivity Kit (Invitrogen) and the DNA integrity was assessed using a Bioanalyzer High Sensitivity Chip (Agilent). The amplified DNA was then used for library preparation.

This procedure and massively parallel sequencing were performed by the Center for Genome Research and Biocomputing (Oregon State University). The DNA libraries were generated, PCR-amplified, and quantified as described above for RNA-seq. The resulting samples were run on a HiSeq 3000 with a targeted density of 300 million reads per lane, and using a single-read 100-cycle protocol. The data were analyzed as outlined above for RNA-seq.

ChIP-seq data analysis. To study chromatin transitions by ChIP-seq analysis, sequences were filtered using Trimmomatic and mapped to the rn6 reference rat genome using the Bowtie2 aligner as described above (RNA-seq data analysis). To identify the regions of the genome enriched in H3K27ac and H3K4me1, we visualized the alignment of mapped reads using the Integrated Genome Viewer ${ }^{53}$ We also utilized $\mathrm{MACS}^{54}$ to identify individual peaks for each epigenomic mark within each sample. We utilized a control based on random shuffling of the original 
alignments rather than input DNA for MACS analysis as such controls have been shown to be nearly as effective as input DNA ${ }^{55,56}$

Fluorescent in situ hybridization. For this procedure we used four LJ 28-day-old female rats. Following intracardiac perfusion with $4 \%$ paraformaldehyde borate buffer, $\mathrm{pH} 9.5$, we processed the brains for hybridization histochemistry, as described $^{57,58}$. We used the double FISH procedure described by Watakabe et al. ${ }^{59}$ employing various complementary (c)RNA probes. Probes complementary to a COMPASS mRNA (Set1b) and COMPASS-like mRNAs (Mll1/KMT2a, Mll2/ $\mathrm{Kmt} 2 b, \mathrm{Mll3} / \mathrm{Km} 2 \mathrm{c}$, and $\mathrm{Mll4} / \mathrm{Kmt} 2 \mathrm{~d}$ ) were labeled with fluorescein-12-UTP (FITC). A Kiss1 cRNA probe was labeled with digoxigenin-11-UTP (Dig). The labeling reactions were performed in a $10 \mu \mathrm{l}$ volume, containing $1 \mu \mathrm{l}$ of a $2 \mathrm{mM}$ digoxigenin-UTP solution; $500 \mathrm{ng}$ of cDNA template; $2.5 \mathrm{mM}$ of each ATP, CTP, and GTP; and 15-20 U of SP6 RNA polymerase. Following $1 \mathrm{~h}$ of incubation at $40^{\circ}$ $\mathrm{C}$ the reaction was treated with DNase, stabilized with dithiothreitol (DTT) and salt, and the volume was adjusted to $100 \mu \mathrm{l}$ with $20 \mathrm{mM}$ DTT. The cRNA probe was precipitated with ethanol, dried, and resolubilized in $100 \mu \mathrm{l}$ diethyl pyrocarbonatetreated water. Five microliters of the mixture were then run on a formaldehyde agarose gel to assess the integrity of the cRNAs and the yield of the reaction, as reported ${ }^{60}$.

Control sections were incubated with sense probes transcribed from the same plasmid, but linearized on the $3^{\prime}$-end to transcribe the coding strand of the cDNA template. Following treatment with proteinase $\mathrm{K}$ and acetic anhydride, the sections were hybridized overnight at $55^{\circ} \mathrm{C}$ with Dig-Kiss 1 cRNA in combination with either FITC-COMPASS mRNA (Set $1 b)$ or FITC-COMPASS-like mRNAs (Mll1/ $K M T 2 a, M l l 2 / K m t 2 b, M l l 3 / K m t 2 c$, and $M l l 4 / K m t 2 d)$. The next day, the slides were washed at high stringency (final wash: $0.1 \times$ sodium chloride-sodium citrate at $65^{\circ}$ $\mathrm{C}$ for $30 \mathrm{~min}$ ). Thereafter, the sections were incubated with $0.3 \% \mathrm{H}_{2} \mathrm{O}_{2}$ for $10 \mathrm{~min}$ to block endogenous peroxidases, followed by $30 \mathrm{~min}$ in $0.5 \%$ blocking reagent provided with the Renaissance tyramine signal amplification (TSA) Plus dinitrophenyl (DNP) system (PerkinElmer, Boston, MA) used to enhanced the FITC reaction. Following these blocking steps, the sections were incubated overnight at $4{ }^{\circ} \mathrm{C}$ simultaneously with antidigoxygenin-alkaline phosphataseconjugated sheep antibodies (Roche, Indianapolis, ID) diluted 1:1000 and a mouse monoclonal anti-FITC antibody conjugated to a peroxidase conjugated IgG fraction (Jackson ImmunoResearch Laboratories, West Grove, PA) diluted 1:4000 in TNT buffer $(0.1 \mathrm{M}$ Tris- $\mathrm{HCl}, 0.15 \mathrm{M} \mathrm{NaCl}$, and $0.5 \%$ Triton X-100). The next day, the sections were washed in TNT buffer (three times, 10 min each) before a 30 min incubation at room temperature with TSA Plus DNP reagent (PerkinElmer) diluted 1:50. Following three washes in TNT buffer ( $10 \mathrm{~min}$ each), the sections were incubated with rabbit anti-DNP-keyhole limpet hemocynin Alexa 488 antibodies (Invitrogen/Molecular Probes, Eugene, OR) diluted in TNT buffer for 2 $\mathrm{h}$ at room temperature to develop the FITC reaction into green fluorescence. Thereafter, the sections were again washes in TNT buffer (three times, $10 \mathrm{~min}$ each time) followed by one wash in TS8.0 buffer ( $0.1 \mathrm{M}$ Tris- $\mathrm{HCl}, \mathrm{pH} 8.0,0.1 \mathrm{M} \mathrm{NaCl}$, and $10 \mathrm{mM} \mathrm{MgCl}_{2}$ ); the digoxigenin reaction was then converted to red fluorescence by incubating the sections with HNPP (2-hydroxy-3-naphtoic acid-2'phenylanilide phosphate)/Fast Red reagent (Roche) for $30 \mathrm{~min}$ at room temperature. After three washes with in PBS-10 mM EDTA, the sections were incubated with Hoechst 33258 (Invitrogen) at $0.1 \mu \mathrm{g} \mathrm{ml}^{-1}$ for $1 \mathrm{~min}$, washed in PBS, and coverslipped with aqueous mounting medium, before fluorescence microscopy examination ${ }^{9}$.

Probes for in situ hybridization. We employed several cRNAs. A Kiss1 cRNA probe was prepared by transcribing a 393 bp rat Kiss 1 cDNA $^{61}$ (nt 1-393 in rat Kiss1 mRNA; accession No. NM_181692.1). A Set1b cRNA probe was generated by transcribing a 535 bp cDNA template (nt 7390-7925 in the coding region of rat Set $1 b$ mRNA; accession No. XR_001836008.1). An Mll1 cRNA was transcribed from a 522 bp cDNA template (nt 596-1118 in rat Mll1 mRNA; accession No. XM_008766179.1). An Mll2 cRNA was transcribed from a 438 bp cDNA fragment (nt 31-469 in rat Mll2 mRNA; accession No. XM_017595318.1). An Mll3 cRNA was transcribed from a 425 bp cDNA template (nt 5009-5434 in rat Mll3 mRNA; accession No. XM_006235840.3). Finally, an Mll4 cRNA was transcribed from a 630 bp cDNA fragment (nt 6753-7383 in rat Mll4 mRNA; accession No. XM_008759254.2).

All cRNA probes were prepared by in vitro transcription of cDNA templates generated by RT-PCR amplification of hypothalamic total RNA. The PCR fragments were cloned into the pGEM-T vector (Promega) and their identity was verified by sequencing. Primer sequences and regions recognized by the cRNA probes used are shown in Supplementary Table 1.

Small hairpin RNAs and lentivirus construct design. To identify shRNA sequences able to reduce $M l l 1$ mRNA expression, sense and antisense 22-mer oligodeoxynucleotides (Supplementary Table 1) encoding four different shRNAs were designed using the online tool https://sispotr.icts.uiowa.edu/sispotr/index. html;jsessionid=245170F2742B69FB408F737CCE6DAC8F. The sense and antisense sequences were incorporated in silico (http://cancan.cshl.edu/cgi-bin/Codex/ Tools.cgi) into a pPRIME sequence to generate 97-mer oligodeoxynucleotides, which we then purchased from ThermoFisher as PAGE-purified reagents. To convert these long oligodeoxynucleotides into double-stranded DNA, we PCRamplified them using pSM2C ${ }^{62}$ forward (5'-GATGGCTGCTCGAGAAGGTATATTGCTGTTGACAGTGAGCG-3') and reverse (5'-GTCTAGAGTCTAGACGAGGCAGTAGGCA-3') primers. The forward primer contains a XhoI restriction site at the end, and the reverse primer contains an EcoRI site (double underlined $)^{62}$. For amplification we utilized $100 \mathrm{ng}$ of the template DNA, $25 \mathrm{pmol}$ of the forward and reverse synthesis primers, and 1 unit of VentR taq polymerase (New England Biolabs, Ipswich, MA). The PCR reaction consisted of $94^{\circ} \mathrm{C}$ for 5 min, 12 cycles of $94^{\circ} \mathrm{C}, 54^{\circ} \mathrm{C}$, and $75^{\circ} \mathrm{C}\left(30 \mathrm{~s}\right.$ each), and a final incubation at $75^{\circ} \mathrm{C}$ for $2 \mathrm{~min}$. The resulting PCR products were $132 \mathrm{bp}$ in length. Following restriction enzyme digestion, the double-stranded DNA fragments, which generate the required hairpin structure ${ }^{62}$, were $110 \mathrm{bp}$. They were ligated into the XhoI-EcoRI sites of the pPRIME microRNA LV vector system, as recommended ${ }^{62}$. The pPRIME vectors contain a RNA polymerase II promoter that directs expression of a marker gene, which in our construct encodes an eGFP transcribed under the control of the cytomegalovirus (CMV) promoter (Supplementary Fig. 7a). To identify the cells transduced by the virus following either in vitro or in vivo infection we detected eGFP by immunohistofluorescence ${ }^{25}$. The eGFP-encoding sequence is located immediately upstream from an artificial miRNA precursor derived from miR-3063. This miRNA sequence contains adjacent XhoI and EcoRI sites (Supplementary Fig. 7a) that allow replacement of the hairpin sequence with shRNAs targeting the mRNA of interest ${ }^{62,64}$. A siRNA generated against firefly luciferase mRNA ${ }^{62}$, but containing nonsense mutations, was used as a control (C). Because of the mutations introduced, this siRNA targets neither mammalian mRNAs nor the firefly luciferase mRNA ${ }^{62}$. After confirming the sequence of each shRNA construct, infective LV particles were prepared by transient co-transfection of the vector plasmid and the packaging plasmids into 293T cells. The virus was concentrated by ultra-centrifugation, and the resulting pellet was resuspended in Hanks balanced salt solution. The packaging and preparation of the virus has been described in detail elsewhere ${ }^{65}$.

Efficiency of LV-MII1-GFP to reduce MII1 expression in vitro. The ability of the shRNA-Mll1 constructs to reduce Mll1 mRNA levels was tested and compared to the control virus (C) using the immortalized R22 hypothalamic cell line (Cedarlane, Burlington, NC). The cells were plated in DMEM medium at 400,000 cells per well using 12-well plates. Twenty-four hours later, the cells were transduced with the viruses at a multiplicity of infection of 5-1. Three days after the infection, transduced cells (identified by their expression of eGFP) were isolated by flow cytometry to produce a pure population of cells. These cells were expanded and replated onto 12-well plates at a density of 300,000 cells per plate. Three days later, total RNA was extracted as described above and Mll1 mRNA levels were determined by qPCR using the primers listed in Supplementary Table 1.

Preparation of AAV-dSaCas9-KRB-sgRNA constructs. All plasmids used to generate a catalytically deactivated (d) Staphylococcus aureus (Sa)Cas9-KRBsgRNA construct were purchased from Addgene (https://www.addgene.org/). We first excised a $3357 \mathrm{bp}$ DNA fragment containing the coding region of dSaCas 9 from plasmid pX603-AAV-CMV::NLS-dSaCas9 (D10A,N580A)-NLS-3xHAbGHpA using AgeI and EcoRI (ThermoFisher) restriction endonucleases. We then removed a DNA fragment containing the coding region of wild-type SaCas9 from pX601-AAV-CMV::NLS-SaCas9-NLS-3xHA-bGHpA;U6::BsaI-sgRNA also using AgeI and EcoRI, and replaced this DNA segment with that encoding dSaCas9. We termed the new vector pX601-AAV-CMV::NLS-dSaCas9-NLS-3xHA-bGHpA;U6: BsaI-sgRNA. Next, we PCR-amplified from pHR-SFFFV-dCas9-BFP-KRAB a 222 bp DNA fragment containing the KRAB domain using In-fusion PCR technology (Takara, Mountain View, CA) and cloned this fragment into the BamHI site that follows the nuclear localization signal (NLS) at the $3^{\prime}$-end of the dSaCas9 sequence in pX-AAV-CMV::NLS-dSaCas9-NLS-3xHA-bGHpA;U6::BsaI-sgRNA (Supplementary Fig. 6). The primers used are shown in Supplementary Table 1. The resulting vector is termed AAV-dSaCas9-KRAB-3xHA-U6::Bsa1-sgRNA. The integrity of each component added to the original pX601-AAV-CMV::NLSSaCas9-NLS-3xHA-bGHpA;U6::BsaI-sgRNA was verified by sequencing. The construct (sg4) found to increase H3K9me3 deposition at the ARC Kiss1 enhancer most efficiently was modified to generate a new control $(\Delta \mathrm{K})$ devoid of the KRAB domain. We used BamHI and EcoRI to remove the KRAB encoding DNA, T4 DNA polymerase (New England Biolabs) to blunt the open ends, and T4 DNA ligase (New England Biolabs) for plasmid re-ligation.

Preparation of sgRNAs. A panel of five sgRNAs targeting the putative Kiss1 enhancer site 1 was designed and cloned. The sgRNAs were designed using an online tool (https://www.deskgen.com/) searching for the PAMs NNGRRT and $\mathrm{NNGRR}^{36}$. Following phosphorylation and annealing of each oligonucleotide set, the double-stranded products were digested with BsaI (Eco31I), and ligated into the BsaI site of AAV-dSaCas9-KRAB-3xHA-U6::Bsa1-sgRNA. After treatment with Plasmid Safe DNA exonuclease (Epicentre, Madison, WI), $2 \mu \mathrm{l}$ of each reaction were used to transform Stbl3 cells (ThermoFisher). The next day, colonies were collected and grown overnight for DNA extraction and sequencing. The identity of all sgRNAs was confirmed by sequencing using a primer (Supplementary Table 1) complementary to the U6 promoter sequence ${ }^{66}$. 
Evaluation of AAV-dSaCas9-KRAB-sgRNA biological activity. To assess the biological activity of these sgRNAs we used Rat1 cells (ATCC CRL-2210). The cells were plated in the medium described under Functional promoter assays at 200,000 cell per well, using 12-well plates (for qPCR) or 400,000 cells per well in 6-well plates (for ChIP assays).

For chromatin extraction, the cells were transfected $24 \mathrm{~h}$ after plating with the AAV-dSaCas9-KRAB-3xHA-U6::Bsa1-sgRNA constructs using Lipofectamine 3000 (Invitrogen) at a ratio $1 \mu \mathrm{g}$ DNA: $3 \mu \mathrm{l}$ Lipofectamine 3000 in $100 \mu$ l Optimem (Invitrogen). Control cells were transfected with either CK or $\Delta \mathrm{K} .48 \mathrm{~h}$ after transfection, the cells were snap-frozen before measuring by ChIP-qPCR the content of $\mathrm{H} 3 \mathrm{~K} 9 \mathrm{me} 3$ and $\mathrm{H} 3 \mathrm{~K} 27 \mathrm{ac}$ at the Kiss 1 enhancer site 1 and Kiss 1 promoter.

For Kiss1, Tac3, and Pdyn mRNA measurements, we treated the cells with 5azacytidine ( $1 \mathrm{nM}$; Sigma-Aldrich) to reduce DNA methylation that may be repressing gene expression ${ }^{67}$. The treatment was initiated $24 \mathrm{~h}$ before transfection and was maintained until the end of the experiment, i.e., $48 \mathrm{~h}$ after transfection. Because the AAV-dSaCas9-KRAB-3xHA-U6::Bsa1-sgRNA constructs we used do not carry a GFP marker, and thus do not allow fluorescent-based cell sorting, we co-transfected each construct $\left(1 \mu \mathrm{g}\right.$ per $1 \times 10^{6}$ cells $)$ with pcDNA-GFP $(0.2 \mu \mathrm{g}$ per $1 \times 10^{6}$ cells; 5:1 ratio). Forty-eight hours after co-transfection, the cells expressing GFP were isolated by FACS, and frozen at $-80^{\circ} \mathrm{C}$ until Kiss1, Tac3, and Pdyn mRNA levels were determined by qPCR.

Detection of hypothalamic LV infection. Cells transduced with C or sh2612 were identified in $35 \mu \mathrm{m}$ brain sections by immunohistofluorescence using a goat polyclonal antibody against eGFP (1:2000, AbCam, Cambridge, MA; Supplementary Table 3). Following an overnight incubation with this antibody, the sections were incubated for $1 \mathrm{~h}$ at room temperature with an Alexa 488 donkey antigoat IgG (1:500, Invitrogen), followed by 1 min incubation with Hoechst 33258 reagent (Thermo Fisher; 1:10,000) to stain cell nuclei.

Detection of dCas9 mRNA in the hypothalamus. To assess the accuracy of the intrahypothalamic injections of sg4 we used qPCR to measure the levels of $d \operatorname{Cas} 9$ mRNA attained in the ARC as compared with the lateral hypothalamus at the time of completion of the study. The primers used are shown in Supplementary Table 1.

Statistics. All statistical analyses were performed using Prism 7.0 software (GraphPad, San Diego, CA). The data were first subjected to a normality and an equal variance test. Data that passed these two tests were then analyzed by either analysis of variance (ANOVA) followed by the Student-Newman-Keuls to compare multiple groups, the Dunnett's test to compare several groups to a single control group, or the Student's $t$-test to compare two groups. When comparing percentages, groups were subjected to an arc-sine transformation before statistical analysis to convert the values from a binomial to a normal distribution ${ }^{6}$. The sample size was selected based on power analyses performed using the s.d.'s that we normally observe when measuring the parameters examined in this study and an $n$ $=6$ per group. These analyses provide at least $80 \%$ (type II error $=0.124$ ) power to detect two effect sizes using either ANOVA or two-sided two-sample $t$-test with a significance level of 0.05 . The investigator was blinded to the group allocation in all physiological and molecular determinations.

Data availability. The RNA-seq and ChIP-seq data have been deposited into the Gene Expression Omnibus hosted at the National Center for Biotechnology Information with the accession numbers GSE94080 and GSE95660 respectively.

Received: 19 July 2017 Accepted: 1 December 2017

Published online: 04 January 2018

\section{References}

1. Boyar, R. et al. Synchronization of augmented luteinizing hormone secretion with sleep during puberty. N. Engl. J. Med. 287, 582-586 (1972).

2. Navarro, V. M. et al. Regulation of NKB pathways and their roles in the control of Kiss1 neurons in the arcuate nucleus of the male mouse. Endocrinology 152, 4265-4275 (2011)

3. Lehman, M. N., Coolen, L. M. \& Goodman, R. L. Minireview: kisspeptin/ neurokinin $\mathrm{B} /$ dynorphin (KNDy) cells of the arcuate nucleus: a central node in the control of gonadotropin-releasing hormone secretion. Endocrinology 151, 3479-3489 (2010).

4. Wakabayashi, Y. et al. Neurokinin B and dynorphin A in kisspeptin neurons of the arcuate nucleus participate in generation of periodic oscillation of neural activity driving pulsatile gonadotropin-releasing hormone secretion in the goat. J. Neurosci. 30, 3124-3132 (2010).
5. Beale, K. E. et al. The physiological role of arcuate kisspeptin neurons in the control of reproductive function in female rats. Endocrinology 155, 1091-1098 (2014).

6. Topaloglu, A. K. et al. Inactivating KISS1 mutation and hypogonadotropic hypogonadism. N. Engl. J. Med. 366, 629-635 (2012).

7. Topaloglu, A. K. et al. TAC3 and TACR 3 mutations in familial hypogonadotropic hypogonadism reveal a key role for Neurokinin B in the central control of reproduction. Nat. Genet. 41, 354-358 (2008).

8. Pinilla, L., Aguilar, E., Dieguez, C., Millar, R. P. \& Tena-Sempere, M. Kisspeptins and reproduction: physiological roles and regulatory mechanisms Physiol. Rev. 92, 1235-1316 (2012).

9. Lomniczi, A. et al. Epigenetic control of female puberty. Nat. Neurosci. 16, 281-289 (2013).

10. Simon, J. A. \& Kingston, R. E. Mechanisms of polycomb gene silencing: knowns and unknowns. Nat. Rev. Mol. Cell Biol. 10, 697-708 (2009).

11. Schwartz, Y. B. \& Pirrotta, V. Polycomb silencing mechanisms and the management of genomic programmes. Nat. Rev. Genet. 8, 9-22 (2007).

12. Piunti, A. \& Shilatifard, A. Epigenetic balance of gene expression by Polycomb and COMPASS families. Science 352, aad9780 (2016)

13. Schuettengruber, B., Martinez, A. M., Iovino, N. \& Cavalli, G. Trithorax group proteins: switching genes on and keeping them active. Nat. Rev. Mol. Cell Biol. 12, 799-814 (2011).

14. Shilatifard, A. The COMPASS family of histone H3K4 methylases: mechanisms of regulation in development and disease pathogenesis. Annu. Rev. Biochem. 81, 65-95 (2012).

15. Berger, S. L. The complex language of chromatin regulation during transcription. Nature 447, 407-412 (2007).

16. Wang, Z. et al. Combinatorial patterns of histone acetylations and methylations in the human genome. Nat. Genet. 40, 897-903 (2008).

17. Denissov, S. et al. Mll2 is required for $\mathrm{H} 3 \mathrm{~K} 4$ trimethylation on bivalent promoters in embryonic stem cells, whereas Mll1 is redundant. Development 141, 526-537 (2014).

18. $\mathrm{Wu}, \mathrm{M}$. et al. Molecular regulation of $\mathrm{H} 3 \mathrm{~K} 4$ trimethylation by Wdr82, a component of human Set1/COMPASS. Mol. Cell Biol. 28, 7337-7344 (2008).

19. Hu, D. et al. The MLL3/MLL4 branches of the COMPASS family function as major histone H3K4 monomethylases at enhancers. Mol. Cell Biol. 33, 4745-4754 (2013).

20. Tie, F. et al. Trithorax monomethylates histone $\mathrm{H} 3 \mathrm{~K} 4$ and interacts directly with CBP to promote H3K27 acetylation and antagonize Polycomb silencing. Development 141, 1129-1139 (2014).

21. Bernstein, B. E. et al. A bivalent chromatin structure marks key developmental genes in embryonic stem cells. Cell 125, 315-326 (2006).

22. Krishnan, S., Horowitz, S. \& Trievel, R. C. Structure and function of histone H3 lysine 9 methyltransferases and demethylases. ChemBioChem. 12, 254-263 (2011).

23. Mueller, J. K. et al. Transcriptional regulation of the human KiSS1 gene. Mol. Cell Endocrinol. 342, 8-19 (2011).

24. Ha, C. M. et al. NELL2, a neuron-specific EGF-like protein, is selectively expressed in glutamatergic neurons and contributes to the glutamatergic control of GnRH neurons at puberty. Neuroendocrinology 88, 199-211 (2008).

25. Heger, S. et al. Enhanced at puberty 1 (EAP1) is a new transcriptional regulator of the female neuroendocrine reproductive axis. J. Clin. Invest. 117, 2145-2154 (2007).

26. Zhou, J. X. et al. Combined modulation of polycomb and trithorax genes rejuvenates beta cell replication. J. Clin. Invest. 123, 4849-4858 (2013).

27. Goto, T. et al. Identification of hypothalamic arcuate nucleus-specific enhancer region of Kiss1 gene in mice. Mol. Endocrinol. 29, 121-129 (2015).

28. Tomikawa, J. et al. Epigenetic regulation of Kiss1 gene expression mediating estrogen-positive feedback action in the mouse brain. Proc. Natl Acad. Sci. USA 109, E1294-E1301 (2012).

29. Kim, T. K. \& Shiekhattar, R. Architectural and functional commonalities between enhancers and promoters. Cell 162, 948-959 (2015).

30. Kim, T. K. et al. Widespread transcription at neuronal activity-regulated enhancers. Nature 465, 182-187 (2010).

31. Herz, H. M. et al. Enhancer-associated H3K4 monomethylation by Trithoraxrelated, the Drosophila homolog of mammalian Mll3/Mll4. Genes Dev. 26, 2604-2620 (2012).

32. Heintzman, N. D. et al. Distinct and predictive chromatin signatures of transcriptional promoters and enhancers in the human genome. Nat. Genet. 39, 311-318 (2007).

33. Thakore, P. I. et al. Highly specific epigenome editing by CRISPR-Cas 9 repressors for silencing of distal regulatory elements. Nat. Methods 12, 1143-1149 (2015).

34. Kearns, N. A. et al. Functional annotation of native enhancers with a Cas9histone demethylase fusion. Nat. Methods 12, 401-403 (2015).

35. Thakore, P. I., Black, J. B., Hilton, I. B. \& Gersbach, C. A. Editing the epigenome: technologies for programmable transcription and epigenetic modulation. Nat. Methods 13, 127-137 (2016). 
36. Ran, F. A. et al. In vivo genome editing using Staphylococcus aureus Cas9. Nature 520, 186-191 (2015).

37. Ying, Y. et al. The Kruppel-associated box repressor domain induces reversible and irreversible regulation of endogenous mouse genes by mediating different chromatin states. Nucleic Acids Res. 43, 1549-1561 (2015).

38. Seminara, S. B. et al. The GPR54 gene as a regulator of puberty. N. Engl. J. Med. 349, 1614-1627 (2003).

39. Wang, Y., Li, X. \& Hu, H. H3K4me2 reliably defines transcription factor binding regions in different cells. Genomics 103, 222-228 (2014).

40. Sims, R. J. III \& Reinberg, D. Histone H3 Lys 4 methylation: caught in a bind? Genes Dev. 20, 2779-2786 (2006).

41. Calo, E. \& Wysocka, J. Modification of enhancer chromatin: what, how, and why? Mol. Cell 49, 825-837 (2013).

42. Bianco, S. D. \& Kaiser, U. B. The genetic and molecular basis of idiopathic hypogonadotropic hypogonadism. Nat. Rev. Endocrinol. 5, 569-576 (2009).

43. Kim, H. G. et al. Mutations in CHD7, encoding a chromatin-remodeling protein, cause idiopathic hypogonadotropic hypogonadism and Kallmann syndrome. Am. J. Hum. Genet. 83, 511-519 (2008).

44. Watanabe, G. \& Terasawa, E. In vivo release of luteinizing hormone releasing hormone increases with puberty in the female rhesus monkey. Endocrinology 125, 92-99 (1989).

45. Ojeda, S. R. \& Skinner, M. K. in The Physiology of Reproduction 3rd edn (ed. Neill, J. D.) 2061-2126 (Academic Press/Elsevier, San Diego, 2006).

46. Urbanski, H. F. \& Ojeda, S. R. The juvenile-peripubertal transition period in the female rat: Establishment of a diurnal pattern of pulsatile luteinizing hormone secretion. Endocrinology 117, 644-649 (1985).

47. Ojeda, S. R. \& Terasawa, E. in Hormones, Brain and Behavior, Vol 4 (eds Pfaff, D. et al.) 589-659 (Elsevier, New York, 2002).

48. Dissen, G. A. et al. Excessive ovarian production of nerve growth factor facilitates development of cystic ovarian morphology in mice and is a feature of polycystic ovarian syndrome in humans. Endocrinology 150, 2906-2914 (2009).

49. Robinson, M. D., McCarthy, D. J. \& Smyth, G. K. edgeR: a Bioconductor package for differential expression analysis of digital gene expression data. Bioinformatics 26, 139-140 (2010).

50. Bolger, A. M., Lohse, M. \& Usadel, B. Trimmomatic: a flexible trimmer for Illumina sequence data. Bioinformatics 30, 2114-2120 (2014).

51. Langmead, B. \& Salzberg, S. L. Fast gapped-read alignment with Bowtie 2. Nat. Methods 9, 357-359 (2012).

52. Kim, D. et al. TopHat2: accurate alignment of transcriptomes in the presence of insertions, deletions and gene fusions. Genome Biol. 14, R36 (2013).

53. Robinson, J. T. et al. Integrative genomics viewer. Nat. Biotechnol. 29, 24-26 (2011).

54. Zhang, Y. et al. Model-based analysis of ChIP-Seq (MACS). Genome Biol. 9 , R137 (2008).

55. Szalkowski, A. M. \& Schmid, C. D. Rapid innovation in ChIP-seq peak-calling algorithms is outdistancing benchmarking efforts. Brief. Bioinform. 12, 626-633 (2011).

56. Zhang, Y. F. \& Su, B. Peak identification for ChIP-seq data with no controls. Dongwuxue. Yanjiu 33, E121-E128 (2012).

57. Simmons, D. M., Arriza, J. L. \& Swanson, L. W. A complete protocol for in situ hybridization of messenger RNAs in brain and other tissues with radiolabeled single-stranded RNA probes. J. Histotechnol. 12, 169-181 (1989).

58. Rage, F., Lee, B. J., Ma, Y. J. \& Ojeda, S. R. Estradiol enhances prostaglandin E2 receptor gene expression in luteinizing hormone-releasing hormone (LHRH) neurons and facilitates the LHRH response to PGE2 by activating a glia-toneuron signaling pathway. J. Neurosci. 17, 9145-9156 (1997).

59. Watakabe, A., Komatsu, Y., Ohsawa, S. \& Yamamori, T. Fluorescent in situ hybridization technique for cell type identification and characterization in the central nervous system. Methods 52, 367-374 (2010).

60. Berg-von der Emde, K. et al. Neurotrophins and the neuroendocrine brain: different neurotrophins sustain anatomically and functionally segregated subsets of hypothalamic dopaminergic neurons. J. Neurosci. 15, 4223-4237 (1995).

61. Mastronardi, C. et al. Deletion of the Ttf1 gene in differentiated neurons disrupts female reproduction without impairing basal ganglia function. $J$. Neurosci. 26, 13167-13179 (2006).
62. Stegmeier, F., Hu, G., Rickles, R. J., Hannon, G. J. \& Elledge, S. J. A lentiviral microRNA-based system for single-copy polymerase II-regulated RNA interference in mammalian cells. Proc. Natl Acad. Sci. USA 102, 13212-13217 (2005).

63. Lagos-Quintana, M., Rauhut, R., Lendeckel, W. \& Tuschl, T. Identification of novel genes coding for small expressed RNAs. Science 294, 853-858 (2001).

64. Silva, J. M. et al. Second-generation shRNA libraries covering the mouse and human genomes. Nat. Genet. 37, 1281-1288 (2005).

65. Dissen, G. A. et al. In vivo manipulation of gene expression in non-human primates using lentiviral vectors as delivery vehicles. Methods 49, 70-77 (2009).

66. Ran, F. A. et al. Genome engineering using the CRISPR-Cas9 system. Nat. Protoc. 8, 2281-2308 (2013).

67. Andrews, D. F. III, Nemunaitis, J., Tompkins, C. \& Singer, J. W. Effect of 5azacytidine on gene expression in marrow stromal cells. Mol. Cell Biol. 9, 2748-2751 (1989).

68. Zar, J. H. Biostatistical Analysis, 2nd edn (Prentice Hall, Englewood Cliffs, NJ, 1984).

\section{Acknowledgements}

This work was supported by grants from the US National Science Foundation (NSF: IOS1121691) to S.R.O., and the National Institute of Health (NIH 1R01HD084542) to S R.O. and A.L., and 8P51OD011092 for the operation of the Oregon National Primate Research Center. C.A.T. was supported by NIH NRSA grant F32-HD-86904. C.A.T. and H.W. were supported by NIH Training grants T32-HD007133 and T32-DK 7680. We thank Ali Shilatifard (Feinberg School of Medicine, Northwestern University, Chicago, IL) for providing us with COMPASS protein antibodies for ChIP assays.

\section{Author contributions}

C.A.T. contributed to the study design, conducted the physiological experiments, analytical procedures, and molecular analyses, evaluated and discussed the data, and helped in preparing the figures; H.W. performed the RNA-seq and CHIP-seq analyses; C.F.A. participated in the studies involving molecular procedures, and contributed to figure and manuscript preparation; S.R.O. and A.L. supervised the study design, analyzed the data and wrote the manuscript, which was revised by the rest of the authors. All the authors take full responsibility for the work.

\section{Additional information}

Supplementary Information accompanies this paper at https://doi.org/10.1038/s41467017-02512-1.

Competing interests: The authors declare no competing financial interests.

Reprints and permission information is available online at http://npg.nature.com/ reprintsandpermissions/

Publisher's note: Springer Nature remains neutral with regard to jurisdictional claims in published maps and institutional affiliations.

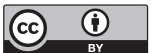

Open Access This article is licensed under a Creative Commons Attribution 4.0 International License, which permits use, sharing, adaptation, distribution and reproduction in any medium or format, as long as you give appropriate credit to the original author(s) and the source, provide a link to the Creative Commons license, and indicate if changes were made. The images or other third party material in this article are included in the article's Creative Commons license, unless indicated otherwise in a credit line to the material. If material is not included in the article's Creative Commons license and your intended use is not permitted by statutory regulation or exceeds the permitted use, you will need to obtain permission directly from the copyright holder. To view a copy of this license, visit http://creativecommons.org/ licenses/by/4.0/.

(C) The Author(s) 2017 